\title{
UNA PROPUESTA DE CRÉDITO TRIBUTARIO AL INGRESO PARA CHILE
}

\author{
Claudio A. Agostini \\ Universidad Adolfo Ibáñez \\ Javiera Selman \\ Universidad de Chile \\ Marcela Perticará \\ Universidad Alberto Hurtado
}

\begin{abstract}
Resumen: En las últimas décadas se ha observado en América Latina una expansión del uso de programas de transferencias condicionadas para combatir la pobreza. Si bien estos programas pueden ser efectivos para mejorar el bienestar de los más pobres en el corto plazo y para asegurar ciertos mí-
\end{abstract}

Claudio A. Agostini. Doctor en Economía, University of Michigan. Profesor Titular, Escuela de Gobierno, Universidad Adolfo Ibáñez, Santiago, Chile. Dirección electrónica: agostini@uahurtado.cl.

Javiera Selman M. Master of Arts in Economics, ILADES-Georgetown University. Investigadora Asociada, Centre for New Development Thinking, Facultad de Economía y Negocios, Universidad de Chile, Santiago, Chile. Dirección electrónica: jselman@fen.uchile.cl.

Marcela Perticará. Doctora en Economía, Universidad de Texas A\&M. Profesora Auxiliar, Facultad de Economía y Negocios, Universidad Alberto Hurtado, Santiago, Chile. Dirección electrónica: mperticara@uahurtado.cl. 
nimos en salud y educación, pueden también desincentivar el empleo, creando así una trampa de pobreza y dependencia en el sistema de bienestar social. En otras regiones del mundo se ha optado por usar el sistema tributario como mecanismo no sólo para redistribuir ingresos, sino que también para implementar políticas sociales. Un buen ejemplo es el Earned Income Tax Credit (EITC) en Estados Unidos, el cual entrega a las personas de menores ingresos un crédito reembolsable condicional en trabajar. Esta política ha logrado simultáneamente aumentar la participación laboral y reducir tanto la pobreza, en especial la de mujeres madres solteras, como la desigualdad. Este trabajo estima mediante simulaciones el efecto que tendría implementar un esquema como el EITC en Chile. Los resultados indican que a través de un crédito tributario es posible aumentar la participación laboral al mismo tiempo que reducir la pobreza y la desigualdad. Adicionalmente, al comparar los resultados con una simulación del Programa Ingreso Ético Familiar, se concluye que el EITC es más efectivo en aumentar los ingresos de las personas que están bajo la línea de la pobreza y tiene un menor costo de transferencia por familia.

Clasificación JEL: H24, H53, I38.

Palabras clave: EITC, pobreza, desigualdad, ingreso ético familiar, Chile.

Recibido: julio 2012; aceptado: noviembre 2012.

\section{A PROPOSAL ON AN INCOME TAX CREDIT FOR CHILE}

Abstract: In recent decades, we have seen in Latin America an increase in the use of conditional cash transfer programs to fight poverty. Although these programs can be effective to improve the welfare of the poor in the short term and to guarantee a certain basic health care and education, they can also discourage employment, thus creating a poverty trap and a dependence on the social welfare system. In other regions of the world, the tax system has been used not only to redistribute income, but also to implement social policies. A good example is the Earned Income Tax Credit (EITC) in the United States, which offers to lower-income individuals a reimbursable credit conditioned on working. This policy has simultaneously increased employment, reduced inequality and reduced poverty particularly among single mothers. This paper estimates, through simulation, the effect that a system 
like the EITC would have in Chile. The results show that a tax credit could increase employment and at the same time reduce poverty and inequality. Additionally, a comparison of the results to a simulation of the Ethical Family Income Program allows concluding that the EITC is more effective in increasing the income of individuals below the poverty line and it has a lower transfer cost per family.

JEL Classification: H24, H53, $I 38$.

Keywords: EITC, poverty, inequality, ethical family income, Chile.

Received: July 2012; accepted: November 2012.

\section{Introducción}

$\mathrm{R}$ educir la pobreza es, en general, uno de los objetivos importantes de cualquier gobierno, en especial en países en vías de desarrollo como Chile. Si bien para ello se considera explícitamente la entrega de subsidios monetarios que directamente aumentan los recursos disponibles en el hogar, aliviando así la pobreza en el corto plazo, hay también políticas de más largo plazo que tienen por objetivo aumentar la capacidad de generación de ingresos autónomos en el hogar. Las políticas de empleo juegan precisamente ese rol mediante programas que buscan mejorar la empleabilidad de las personas y aumentar la probabilidad de encontrar un empleo estable y bien remunerado.

Lamentablemente, en este contexto, algunas políticas de corto plazo pueden tener efectos negativos en las políticas de largo plazo. Por ejemplo, los programas de transferencias condicionadas han mostrado ser efectivos en reducir la pobreza y la desigualdad en el corto plazo, al mismo tiempo que aumentan la matrícula escolar y la asistencia de niños a recintos de salud (Fiszbein y Schady, 2009, Bouillon y Tejerina 2007). No obstante, producen al menos dos efectos negativos que constituyen un dilema importante para efectos de su diseño e implementación. El primero es que desincentivan el empleo, lo que genera un conflicto entre eficiencia y equidad, muchas veces llamado trampa de pobreza. El segundo es que fomentan la dependencia del sistema de bienestar social al no considerar componentes de creación de empleo permanente. Los resultados son aun peores en el caso de los programas 
de transferencias no condicionadas, ya que ni siquiera producen los efectos positivos en la escolaridad y salud de los niños.

En América Latina ha habido en las últimas dos décadas una expansión de programas de transferencias condicionadas. Chile, no ajeno a esta tendencia, implementó en el año 2002 el programa Chile Solidario y recientemente, en mayo de 2012, promulgó la Ley del Programa Ingreso Ético Familiar (IEF), que se basa en transferencias de ingreso condicionadas y no condicionadas, y en un subsidio al empleo de la mujer. Como un primer paso hacia el Ingreso Ético Familiar, en marzo de 2011 comenzó a implementarse el Programa Asignación Social o Bonificación al Ingreso Ético Familiar.

El Programa de Asignación Social consiste en un bono base mensual (incondicional) por individuo y cinco transferencias condicionadas a actividades de salud preventiva, a la matrícula, asistencia y desempeño escolar de los niños del hogar, y a la inserción laboral de mujeres mayores de 18 años. Si bien tiene un componente de creación de empleo, al igual que el Programa Chile Solidario, se basa fundamentalmente en la entrega de un subsidio directo de ingreso no laboral a las familias, lo que reduce los incentivos a trabajar y podría comprometer el objetivo primario del programa, que es reducir la pobreza extrema y mejorar el bienestar permanente de los hogares más pobres del país.

En otras regiones del mundo, como América del Norte, Europa y Oceanía ${ }^{1}$, la estrategia ha sido distinta y se han creado programas que incentivan el empleo al mismo tiempo que alivian la pobreza. Para ello se ha puesto énfasis en el uso del sistema tributario como mecanismo no sólo para redistribuir ingresos sino que también para implementar políticas sociales. Bajo este esquema muchos de los beneficios sociales se entregan en forma de crédito en la devolución anual de impuestos, se reducen las tasas impositivas para grupos de bajos ingresos y/o se eliminan algunos privilegios tributarios a transferencias a desempleados e inactivos (out of work benefits).

Estados Unidos fue pionero en la adopción de este tipo de medidas al establecer en 1975 un sistema de crédito tributario reembolsable focalizado en familias de bajos o moderados ingresos

${ }^{1}$ Específicamente Canadá, Estados Unidos, Austria, Bélgica, Finlandia, Francia, Alemania, Irlanda, Grecia, Italia, Holanda, Inglaterra, Nueva Zelanda y Australia. 
denominado Earned Income Tax Credit (EITC) ${ }^{2}$. La recepción del crédito está sujeta a la condición de trabajar, y si el monto del crédito es mayor que lo que una familia debe pagar en impuestos, entonces se le hace una transferencia monetaria. En la práctica, para las familias de bajos ingresos esto es equivalente a un impuesto negativo.

El crédito tributario al ingreso tiene claras ventajas como mecanismo de redistribución. Ante todo, esta política reduce la pobreza al mismo tiempo que incentiva el trabajo entre los más pobres, disminuyendo el estigma asociado a ser beneficiario de programas sociales. Adicionalmente, tiene menores costos administrativos, debido a que usa la infraestructura e institucionalidad de administración tributaria ya existente en el país, y es eficiente como política tributaria, ya que cuando hay preferencias por redistribución, un impuesto al ingreso óptimo incluye un impuesto negativo (Mirrlees 1971, 1976).

Un sistema de crédito tributario de este tipo es una alternativa viable para disminuir significativamente el conflicto entre corto y largo plazo que producen los programas de transferencias condicionadas. Por esto, y por la inexistencia de programas sociales a través de incentivos tributarios en América Latina y la reciente puesta en marcha del Programa Ingreso Ético Familiar, creemos que es relevante evaluar la implementación de un esquema de crédito tributario al ingreso en Chile.

El resto del trabajo continúa de la siguiente manera. La sección 2 explica los incentivos que genera un crédito tributario al ingreso y la evidencia empírica en la literatura económica respecto a la magnitud de sus efectos. La sección 3 detalla la simulación que se realiza en este trabajo y los datos utilizados para hacerla. La sección 4 presenta los resultados de las simulaciones, con especial foco en sus efectos sobre pobreza y desigualdad. La sección 5 compara la propuesta de EITC de este trabajo con el Programa Ingreso Ético Familiar. Finalmente, la sección 6 presenta conclusiones.

${ }^{2}$ Este modelo se expandió a más países y áreas del sistema de protección social. Además del crédito por la generación de ingresos del trabajo (Work Income Tax Credit) existe el crédito por gastos familiares (Family Tax Credit) y por costos asociados a la primera infancia (Child Care Tax Credit). Algunos ejemplos de países que adoptaron esquemas similares al EITC son Nueva Zelanda (Working for Families Tax Credit) en 1984 e Inglaterra (Working Tax Credit) en 1999. 


\section{Los incentivos del mecanismo de crédito tributario al ingreso}

Un programa de crédito tributario al ingreso incentiva el empleo y contribuye a la reducción de la pobreza y la desigualdad. Ello ocurre a partir de sus efectos en la expansión de la restricción presupuestaria de las personas, el cambio en los precios relativos de la decisión entre ocio y consumo y la variación en la tasa marginal de impuesto que enfrentan los contribuyentes.

El programa de crédito tributario al ingreso más estudiado en la literatura económica ha sido el Earned Income Tax Credit (EITC) implementado en Estados Unidos. Si bien en sus inicios (1975) fue un programa relativamente modesto, experimentó consecutivas expansiones en los años 1986, 1991 y 1993, la mayor de las cuales fue en este último año.

GRÁFICO N ${ }^{\circ} 1: \quad$ EFECTO DEL EITC EN LA RESTRICCIÓN PRESUPUESTARIA

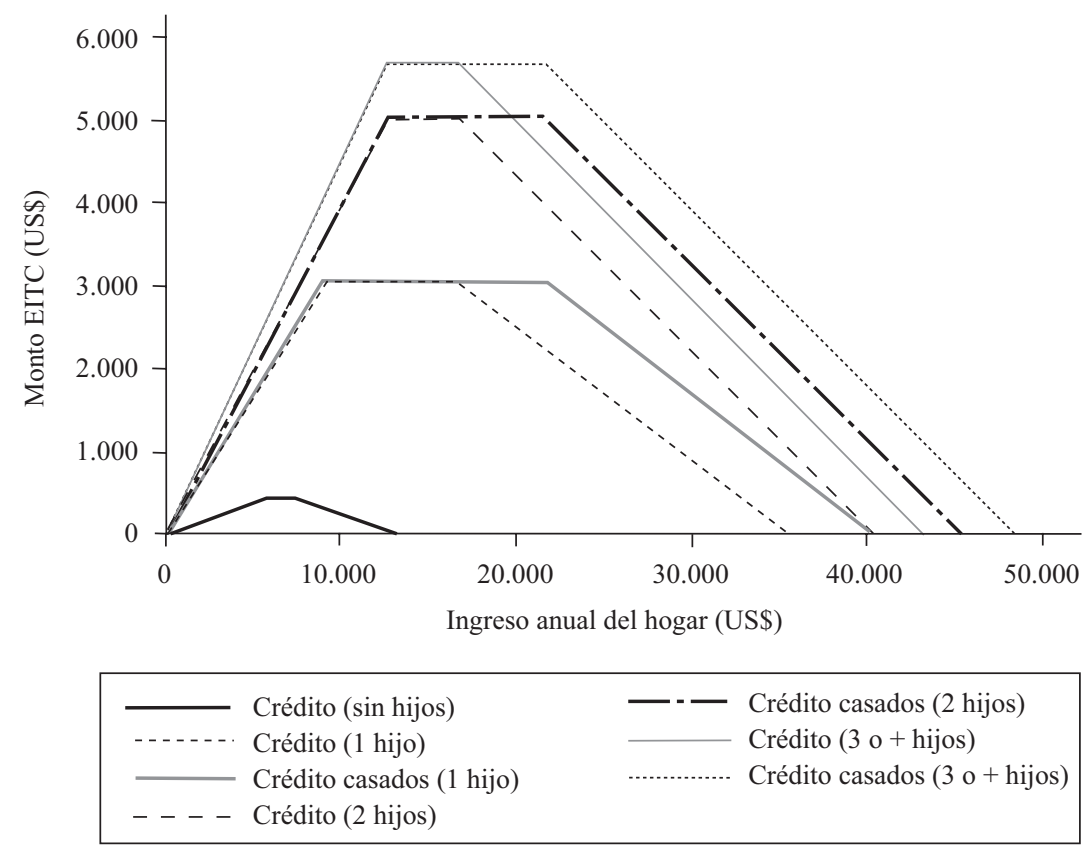

Fuente: Elaboración propia en base a montos 2009 de EITC de Estados Unidos. 
En el EITC el monto del crédito se calcula en base al mayor de los siguientes ingresos: el ingreso del trabajo y, a condición de que la persona trabaje, el ingreso total imponible ${ }^{3}$. Si el crédito supera a lo que debe pagar por impuestos, entonces la familia recibe una transferencia a través de la devolución anual de impuestos. Existe también la opción de recibir un pago mensual, pero ésta es utilizada por una fracción muy pequeña de los beneficiarios.

Los montos y topes del crédito tributario que la familia o individuo reciben dependerán finalmente del nivel de ingreso, el número de hijos elegibles y de si la familia es monoparental o no. Los hijos elegibles son aquellos menores de 18 años, entre 19 y 23 años en caso de que se encuentren estudiando, y niños discapacitados que vivan con el contribuyente más de la mitad del año.

Existen tres segmentos de ingreso en la estructura del EITC para otorgar beneficios: primer segmento (phase in), fase plana (flat region) $\mathrm{y}$ tercer segmento (phase out). En el primero, el EITC es equivalente a un subsidio proporcional al salario, en el segundo es un monto fijo y en el tercero, el crédito es inversamente proporcional al ingreso, decreciendo hasta desaparecer. Las parejas contribuyentes tienen acceso a mayores límites de ingresos en el segundo y tercer segmento. El Gráfico $\mathrm{N}^{\circ} 1$ muestra el esquema del EITC con los montos establecidos para el año 2009 en Estados Unidos.

Los incentivos que genera la aplicación de un esquema como el EITC se pueden analizar a partir del cambio que se produce en la restricción presupuestaria de una persona o familia. El Gráfico $\mathrm{N}^{\circ} 2$ muestra los efectos del EITC sobre la restricción presupuestaria de una familia monoparental (o con un solo trabajador). El eje X son las horas destinadas a trabajo/ocio ${ }^{4}$ y el eje $\mathrm{Y}$ es el consumo o ingreso ${ }^{5}$. La restricción presupuestaria antes del EITC refleja la situación de una persona que está exenta de pagar impuestos.

Con la aplicación del crédito tributario, la restricción presupuestaria aumenta fuertemente en el primer segmento, donde se subsidia el

3 También existe un monto máximo permitido de ingreso por inversiones.

${ }^{4}$ Las horas de ocio crecen desde el origen hacia la derecha. Cuando hay máximo ocio, hay cero horas de trabajo. Por lo tanto, mientras más cerca del origen, más horas de trabajo ofrece la persona o familia.

${ }^{5}$ En estricto rigor, una vez aplicado el EITC se observa el ingreso después de impuesto. 


\section{GRÁFICO N²: $\quad$ EFECTO DEL EITC EN LA RESTRICCIÓN PRESUPUESTARIA}

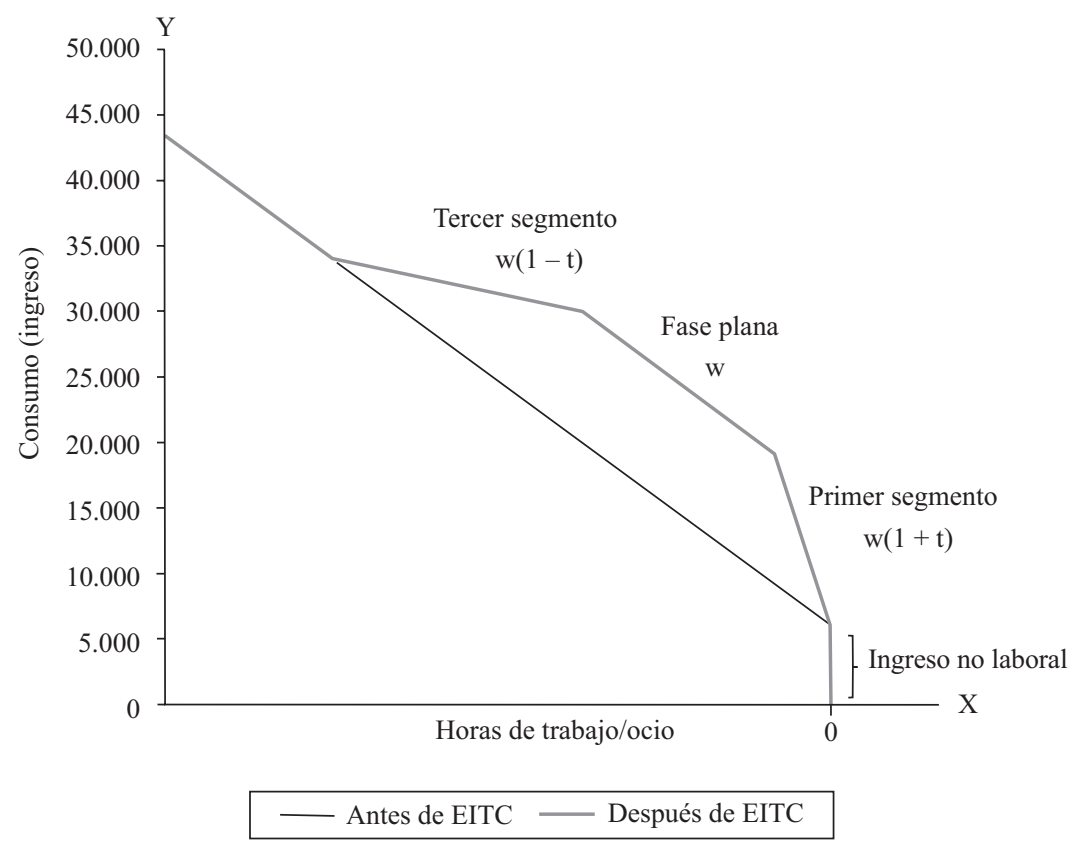

Fuente: Elaboración propia.

salario. Luego, en el segundo segmento, la restricción presupuestaria es paralela a la inicial al aplicarse un subsidio de monto fijo $\mathrm{y}$, finalmente, en la última fase la restricción presupuestaria se va acercando a la inicial al otorgarse un subsidio decreciente al ingreso. Lo relevante es que, tal como se observa en el gráfico, el EITC hace que el ingreso sea siempre mayor o igual a la situación previa al programa, contribuyendo de esta forma a reducir la pobreza y la desigualdad.

A modo de comparación, un programa de transferencias condicionadas (CCT) expande exclusivamente el ingreso no laboral, y el desplazamiento se produce de forma paralela a la restricción presupuestaria inicial, lo que permite que un individuo que no trabaja alcance, con las mismas cero horas de trabajo, un mayor nivel de bienestar. Si el individuo trabaja inicialmente, la expansión de la restricción presupuestaria 
tendrá asociado un efecto ingreso ${ }^{6}$ que le permitirá aumentar sus horas de ocio y su nivel de consumo, es decir, podrá acceder al mismo consumo con un menor número de horas trabajadas.

Si en cambio se utiliza un EITC, la expansión de la restricción presupuestaria no es lineal y esto hace que los incentivos que se generan sobre la oferta laboral varíen dependiendo del segmento en el cual estén los ingresos de las personas, ya que el precio relativo entre ocio y consumo, reflejado en la pendiente de la restricción presupuestaria, va a ser distinto en cada segmento.

Una persona que no trabaje antes ni después del EITC se mantendrá en la situación inicial, pero si un individuo que no trabaja antes se inserta en el mercado laboral, siempre accederá a un nivel de bienestar superior, generando así incentivos a trabajar. Por lo tanto, el efecto en la participación laboral es siempre positivo sobre padres/madres solteros o integrantes de familias de bajos ingresos donde nadie trabaja.

Para una persona que ya trabaja al momento de implementarse el EITC, los incentivos son ambiguos respecto a las horas trabajadas. En el primer tramo, el impacto depende de la magnitud del efecto ingreso (negativo) y el efecto sustitución (positivo). En la fase plana sólo hay efecto ingreso (negativo) y en el tercer segmento ambos efectos son negativos. Adicionalmente, quienes están por encima, pero cerca del límite del tercer segmento, podrían querer reducir las horas trabajadas para ser elegibles (Eissa y Liebman (1996)).

En resumen, se observa que ambas medidas pueden mejorar el bienestar de las personas. Sin embargo, para un mismo nivel de bienestar final, el EITC incentiva el trabajo mientras que un programa de transferencias condicionadas lo desincentiva ${ }^{7}$.

La magnitud del efecto que tiene un EITC en la participación laboral depende de la elasticidad de participación y la demanda de trabajo. A su vez, el efecto en las horas trabajadas está sujeto a la capacidad

${ }^{6}$ El cambio en el ingreso total que se produce cuando cambia el precio relativo entre el ocio y el consumo se puede dividir en un efecto ingreso y un efecto sustitución. El efecto ingreso se refiere a que debido al aumento en el ingreso las personas pueden consumir simultáneamente más ocio y más bienes de consumo, disminuyendo entonces las horas de trabajo. Por otro lado, el efecto sustitución se refiere a que por el mayor precio del ocio las personas van a preferir consumir menos ocio y por lo tanto aumentan las horas de trabajo.

${ }^{7}$ El Gráfico $\mathrm{N}^{\circ}$ A1 del Anexo ilustra la comparación entre estos dos tipos de política. 
de ajustar las horas ofrecidas y a la elasticidad de la oferta laboral. Entonces, el efecto final del EITC dependerá de la cantidad de beneficiarios en cada segmento y de la capacidad de respuesta de los individuos ante esos incentivos (flexibilidad laboral, características de la demanda laboral, nivel del salario mínimo, etc. $)^{8}$. Teóricamente se esperaría un aumento en la participación laboral de los beneficiarios que no estaban insertos en el mercado laboral y un efecto ambiguo o negativo en la cantidad de horas trabajadas de quienes ya trabajaban. La magnitud de estos efectos es una pregunta empírica y la evidencia existente para el programa EITC en Estados Unidos puede iluminar los efectos esperados para Chile.

Existe consenso en la literatura empírica respecto a que el EITC tiene un impacto positivo en los ingresos y ha sido exitoso en reducir la pobreza. En Estados Unidos, por ejemplo, en el año 1999 la brecha agregada de pobreza habría sido $20 \%$ superior de no haber existido el crédito y en el año 2003 cerca de 4,4 millones de personas, de familias con al menos un trabajador, dejaron de ser pobres, siendo la mitad de éstas niños (Holt 2006).

La contribución del EITC va más allá de reducir la pobreza, al disminuir también la dependencia del sistema de bienestar social. Es así como en 1994 había 5 millones de personas afiliadas a algún programa social en Estados Unidos y en 1999 esta cifra había caído en 48\%. En este contexto, los trabajos empíricos indican que las expansiones del EITC ocurridas desde 1986 disminuyeron en 10\% las listas de beneficiarios de programas sociales (Grogger 2003), lo que está asociado a una rebaja importante en el gasto público ${ }^{9}$.

Respecto a los efectos en la oferta laboral, existe una extensa literatura que ha evaluado los efectos del EITC tanto sobre la participación laboral como sobre las horas trabajadas. En Estados Unidos

${ }^{8}$ Un programa como el EITC no cubre a individuos que estén trabajando de manera informal, que no registren ningún tipo de ingresos en el SII, pero es un programa que, en el mediano y largo plazo, hace más atractivo el formalizarse. Sin embargo, en la transición del corto al largo plazo, es necesario mantener programas de asistencia social tradicionales para familias de alta vulnerabilidad, los que podrían ir acompañados de incentivos para la formalización y podrían ser la puerta de entrada al EITC.

${ }^{9}$ Considerando sólo las transferencias del EITC, 250 mil familias que dejan de participar en programas sociales equivalen a un ahorro de mil millones de dólares (Dickert, Hauser y Scholz 1995). 
al mismo tiempo que se expandía el crédito tributario - entre 1986 y 2001 - la tasa de empleo de mujeres madres solteras, que han sido tradicionalmente las principales beneficiarias del EITC, tuvo un alza cercana al 12\% (entre 1984 y 2003). La mayor variación ocurrió entre 1992 y 1999 con un aumento de 16\%. Durante el mismo período se observaron variaciones muy pequeñas en la tasa de empleo de mujeres solteras sin hijos y mujeres casadas con o sin hijos (Eissa y Hoynes 2006). En este contexto, el desafío en esta literatura ha sido identificar hasta qué punto la expansión del EITC pudo haber generado este fuerte aumento en la tasa de participación laboral.

Independientemente de la estrategia de estimación elegida en cada estudio, la evidencia acumulada en la literatura económica concluye en forma robusta que efectivamente el crédito tributario ha sido el principal determinante del aumento en la tasa de empleo de mujeres solteras descrito previamente. Buenos ejemplos de esta evidencia son el trabajo de Meyer y Rosembaum (1999), que a través de técnicas cuasi experimentales encuentran que el EITC explica un tercio del incremento en la tasa de empleo observado entre 1992 y 1996, y el trabajo de Keane y Moffit (1998), que estimando un modelo estructural concluyen que entre 1984 y 1996 el EITC aumentó la participación laboral de madres solteras en $10,7 \%$. No hay evidencia robusta, sin embargo, respecto al impacto que ha tenido el crédito tributario sobre las horas trabajadas, encontrándose efectos nulos o efectos positivos muy pequeños ${ }^{10}$.

Para las mujeres casadas, los pocos estudios disponibles muestran que tanto la participación laboral como las horas trabajadas se reducen producto del EITC. Eissa y Hoynes (2004), por ejemplo, encuentran que la expansión del EITC de 1993 redujo la tasa de participación de mujeres casadas en 1\%. En 2006, los mismos autores a través de una estimación con variables instrumentales, muestran que las expansiones en el período 1986-1996 provocaron una caída de 1 a 4 puntos porcentuales en las

${ }^{10}$ Eissa y Liebman (1995), a partir de técnicas cuasi experimentales, encuentran un aumento en las horas trabajadas de madres solteras en general y un efecto nulo en aquellas con bajo nivel de educación; Keane y Moffit (1998), utilizando un modelo estructural, concluyen que se produce un aumento de $1,4 \%$ en las horas agregadas, aunque no diferencian entre el efecto de las nuevas participantes y de quienes se encontraban trabajando. Resultados similares se encuentran en Meyer y Rosembaum (1999) y Rothstein (2005). 
horas trabajadas de este grupo ${ }^{11}$. La explicación para estos resultados, consistente con la explicación de los incentivos que genera el EITC al desplazar no linealmente la restricción presupuestaria, tiene relación con la ubicación inicial de las beneficiarias en los distintos tramos del EITC. Las mujeres solteras se encuentran principalmente en el tramo inicial phase in, donde domina el efecto sustitución, incrementándose el número de horas trabajadas. Las mujeres casadas, en cambio, se ubican inicialmente en el tramo final phase out, donde prevalece el efecto ingreso, que al ser negativo, reduce las horas trabajadas y la participación laboral.

La sistemática evidencia de efectos significativos sobre participación laboral y efectos pequeños o nulos en las horas trabajadas es consistente con las estimaciones que existen de elasticidad de oferta laboral, las cuales muestran una elasticidad horas-salario mayor para mujeres - especialmente casadas - que para hombres, y considerablemente menor que la elasticidad de participación ${ }^{12}$.

Lo anterior tiene implicancias importantes en el análisis de bienestar. Sáez (2002) muestra que si la elasticidad de participación es alta, el programa de transferencia óptimo ${ }^{13}$ se parece al EITC, con tasas marginales negativas en la parte baja de la distribución del ingreso. En cambio, si las decisiones de las personas fueran fundamentalmente sobre el número de horas ofrecidas, un sistema de impuestos óptimos se acercaría a un Negative Income Tax (NIT), que a diferencia del EITC es un impuesto negativo no condicionado.

La aplicación de un crédito tributario también modifica la tasa marginal de impuesto que pagan los contribuyentes. Este efecto varía según el segmento del crédito en el que se ubique la persona: en la primera fase la tasa marginal cae, siendo incluso negativa para las personas de menores ingresos; luego, en la fase plana, permanece constante; y finalmente en el tercer tramo aumenta ${ }^{14}$. Estos efectos son relevantes

${ }^{11}$ Es importante destacar que no fue sino hasta el año 2001 que se aumentaron los límites de ingreso para las parejas contribuyentes en el segundo y tercer tramo.

12 Véase Triest (1990); Evers, De Mooij y Van Vuuren (2005) y Hotz y Scholz (2003).

${ }^{13}$ La optimalidad se refiere al esquema de tasas marginales que minimiza el exceso de carga tributaria (i.e. la ineficiencia que genera en la asignación de recursos la utilización de impuestos distorsionadores).

14 Por ejemplo, un contribuyente con dos hijos en el tercer segmento que gana un dólar más, deja de recibir 21,06 centavos de crédito, por lo que su tasa marginal aumentó en 21,06\% (Congressional Budget Office 2005). 
para determinar el impacto en la desigualdad. Es así como Wu, Perloff y Golan (2006) estudian el efecto de distintos programas públicos en la distribución del ingreso y muestran que las intervenciones que alteran la tasa marginal de impuesto son efectivas en reducir la desigualdad de ingreso después de impuesto. En este sentido, el EITC juega un rol importante como mecanismo redistributivo entre los programas existentes en Estados Unidos, ya que la evidencia es que los programas de transferencias condicionadas y el salario mínimo no tienen mayor impacto sobre la desigualdad e incluso pueden tener un efecto negativo al no generar los incentivos correctos y tener problemas de focalización.

Por último, la implementación del EITC podría impactar el salario de equilibrio en la economía. Hay dos razones para ello. En primer lugar, los nuevos trabajadores expanden la oferta laboral provocando una presión a la baja. En segundo lugar, un subsidio al ingreso laboral podría hacer que los empleadores bajen los salarios, pudiendo pagar menos por el mismo trabajo. En la práctica, la ocurrencia en magnitudes relevantes de estos dos efectos depende de la flexibilidad salarial y de la política de salario mínimo, el cual reduce la presión a la baja y limita los beneficios que puedan obtener los empleadores de un esquema como el EITC (Eissa y Nichols 2005).

La evidencia para Estados Unidos al menos muestra que las expansiones al EITC ocurridas en los años 90 han tenido un efecto nulo o positivo en las remuneraciones ${ }^{15}$. En los últimos años, sin embargo, cuestionando los trabajos anteriores que no consideraban la incidencia del impuesto sobre el empleador, han aparecido trabajos que buscan evaluar directamente el efecto de la política sobre el salario de equilibrio. Rothstein (2009), en particular, muestra que un dólar de EITC se traduce en una transferencia de US\$0,70 a los beneficiarios y US $\$ 0,73$ a los empleadores de capital humano de baja calificación. Esta diferencia proviene de beneficiarios y no beneficiarios, con quienes compiten los primeros al ingresar al mercado laboral.

Por su parte, Leigh (2010) estima que un aumento del 10\% en el EITC está relacionado con una caída de $2 \%$ en los salarios de quienes tienen educación media completa, $5 \%$ en personas con educación media incompleta y no se percibe ningún efecto para quienes alcanzan educa-

${ }^{15}$ Neumark y Wascher (2001) y Meyer (2007) encuentran que el EITC aumentó el salario de las personas más pobres. Véase también Eissa y Nichols (2005). 
ción superior. No obstante, el autor destaca que en general los estudios muestran que el ingreso total de los más pobres aumenta, revelando que se compensa la caída en los salarios y se logra un mayor ingreso familiar a partir del EITC.

\section{Método de simulación}

Siguiendo la metodología utilizada por Eissa y Hoynes (2008) y Eissa, Kleven y Kreiner (2008), la simulación de la implementación de un crédito tributario al ingreso para Chile consiste en el desarrollo de cuatro etapas consecutivas. En la primera etapa se calcula el ingreso imponible y el monto de impuesto a la renta que debe pagar cada individuo. Para predecir el ingreso laboral de quienes no trabajan al momento de aplicar el EITC, se estima una ecuación del logaritmo del salario por hora para toda la población a través de la estimación propuesta por Heckman $(1979)^{16}$. Los resultados de esta primera etapa de la simulación se muestran en las Tablas Nos. A1 y A2 del Anexo.

En la segunda etapa, se calcula el monto del crédito anual que les corresponde a todas las personas elegibles, es decir, a todos quienes cumplen con los requisitos de edad, hijos e ingresos, independiente de si trabajan o no inicialmente. Dado que la base de datos utilizada, la cual se describe más adelante, cuenta con información de ingreso mensual, se asume que las personas mantendrán sus ingresos por los siguientes doce meses $^{17}$. Para quienes no trabajan antes de la implementación del EITC, pero se insertan al mercado laboral luego de implementado el programa, se simulan dos escenarios alternativos: que se incorporan al mercado laboral trabajando 20 o 45 horas a la semana.

En la tercera etapa, con el ingreso fina ${ }^{18}$ y la variación porcentual respecto al ingreso inicial que significa la recepción del EITC para los elegibles, se identifica a los beneficiarios del programa. Todas las

${ }^{16}$ La predicción del salario por hora para personas que no trabajan supone una distribución del error con media cero y varianza igual a la de la distribución del error de estimación de quienes trabajan.

${ }^{17}$ El 67\% de las personas que cumplen con los criterios de selección para recibir el EITC tiene empleos con fecha de inicio en 2008 o antes.

${ }^{18}$ Es la suma del ingreso después de impuesto, el monto de EITC y las transferencias del Estado (se descuentan los beneficios de desempleo a quienes se insertan al mercado laboral con la medida). 
mujeres elegibles que trabajan inicialmente serán beneficiarias. Para las elegibles que no trabajan inicialmente, según el nivel de la elasticidad de participación laboral y la variación en el ingreso, se calcula el cambio en la probabilidad de participación laboral después de aplicar el EITC $^{19}$. Si la nueva probabilidad es mayor que 0,5 , entonces se asume que la persona decide trabajar y se convierte en beneficiaria. Un supuesto adicional que se hace es que todas las mujeres que deciden trabajar logran encontrar empleo. Si esto ocurre no se sabe qué pasará con los salarios, aunque probablemente una fracción importante tendrá el salario mínimo, lo que frenaría una presión a la baja que pudiera ocurrir ante el aumento en la oferta laboral.

Debido a que no existen estimaciones de la elasticidad de participación laboral en Chile, siguiendo a Eissa y Hoynes (2008), se utilizan cuatro valores para este parámetro: $0 ; 0,1 ; 0,2 ; 0,3^{20}$. Éstos son valores bajos de elasticidad de participación, por lo que los resultados de las simulaciones son conservadores respecto al potencial impacto que puede tener la implementación de un EITC en Chile ${ }^{21}$.

Sin duda, en el inicio de su implementación el EITC podría alterar las horas trabajadas de quienes se encuentran inicialmente trabajando. Si la elasticidad de oferta laboral es $\varepsilon_{\mathrm{k}}$, el número de horas trabajadas para cada individuo después de implementar el EITC se determina por

$$
\operatorname{Horas}_{\text {EITC }}=\operatorname{Horas}_{0}\left(1+\varepsilon_{\mathrm{k}} \cdot \text { variación \% ingreso laboral }\right) \text {. }
$$

Si bien éste es un efecto que potencialmente habría que tomar en cuenta en la simulación, hay autores como Triest (1990) y Meyer (2002) que señalan que la oferta laboral, sujeta a la participación, es

${ }^{19} \operatorname{Prob}\left(S_{\text {EITC }}=1\right)=\Phi\left(Z_{i} \gamma\right)+\phi\left(Z_{i} \gamma\right) \cdot \gamma_{k} \cdot \log ($ cambio ingreso laboral $)$. Donde el cambio en el ingreso laboral es la suma del monto de remuneración mensual predicho y el monto recibido por EITC, descontando los beneficios de desempleo que deja de recibir al insertarse.

${ }^{20}$ Es relevante notar que los valores aquí asumidos son independientes del modelo de salarios con sesgo de selección estimado en la primera etapa. El objeto de la estimación de la ecuación de selección es estimar consistentemente el ingreso laboral predicho de quienes no trabajan al momento de aplicar el EITC. Adicionalmente, a partir de esta primera etapa se obtiene la probabilidad de participación inicial en el mercado laboral de quienes no trabajan antes del EITC.

${ }^{21}$ Rafael de Hoyos (2006) estima para México una participación laboral que fluctúa entre 0,33 (con datos de 1998) y 0,39 (con datos de 2000). 
bastante inelástica y que el EITC afecta únicamente la participación laboral. Meyer (2002) no encuentra ninguna evidencia de que este programa afecte las horas trabajadas de mujeres madres solteras y señala, además, que el asumir la existencia de una elasticidad positiva y uniforme a lo largo de la distribución puede sesgar (sobrestimar) los resultados de una simulación ${ }^{22}$. Por esta razón y por el hecho de que la correcta identificación del efecto sobre las horas trabajadas requiere de distintos supuestos de elasticidad de oferta laboral en los distintos tramos del EITC, en la simulación se asume una elasticidad no compensada de oferta laboral igual a cero $\left(\varepsilon_{k}=0\right)$. Posteriormente, para analizar la robustez de los resultados de las simulaciones se incorpora el supuesto de $\varepsilon_{k}=0.1$.

Finalmente, en la cuarta etapa de la simulación se construye la nueva distribución del ingreso después de impuesto para identificar el efecto del EITC en la participación laboral, la pobreza y la desigualdad.

\section{Datos}

Los datos utilizados en las simulaciones provienen de la Encuesta Casen 2009. De las 246.924 observaciones iniciales se eliminaron las familias o núcleos de servicio doméstico puertas adentro, ya que no viven con sus hijos, y también a aquellos núcleos en que haya personas que trabajan pero no declaran ingresos laborales y/o no tienen horas de trabajo reportadas. Se distingue entre hogar y núcleo (o familia) porque en un hogar con más de un núcleo puede haber más de un beneficiario del EITC. Luego de este proceso quedan 232.772 observaciones que son las que se utilizan para la simulación.

La Tabla $\mathrm{N}^{\circ} \mathrm{A} 3$ del Anexo contiene estadísticas descriptivas de la submuestra utilizada en la simulación y de la muestra completa de la Encuesta Casen 2009. No se observan grandes diferencias entre ambas: el 20\% de los datos corresponde a personas que viven en la Región Metropolitana y el $64 \%$ a residentes de zonas urbanas. El 60\% de la muestra son personas entre 25 y 60 años, la mitad de los encuestados son solteros y en promedio tienen 9 años de educación. El ingreso familiar

${ }^{22}$ Un explicación es la dificultad que pueden tener los beneficiarios para entender la estructura del EITC. Ver Holt (2006) y Chetty y Sáez (2009). 
promedio per cápita antes de impuesto es cercano a $\$ 125.500 \mathrm{y}$, entre los que trabajan, el ingreso del trabajo por hora es en promedio $\$ 2.300$.

Las beneficiarias potenciales de la implementación de un programa como el EITC en Chile son mujeres de 18 a 60 años de edad, de los primeros seis deciles de ingreso, que declaran no ser casadas ni estar conviviendo con una pareja y tienen 0, 1, o 2 o más hijos elegibles. Estos últimos pueden ser menores de 18 años, jóvenes entre 19 y 23 años que estén estudiando, o niños discapacitados que vivan con la beneficiaria.

Entre las mujeres que cumplen con estas características se distinguen dos tipos de beneficiarias: mujeres solteras jefas de familia donde existe al menos un niño elegible y mujeres solteras en familias donde no hay niños elegibles. Esto último se refiere a mujeres mayores de 18 años que no tienen pareja, no estudian, no están discapacitadas y no son elegibles de otro miembro de la familia. En estos casos podría haber más de un beneficiario del programa por núcleo familiar.

La elección de los límites de ingreso, tasas y montos del esquema impositivo está directamente relacionada con el impacto que pueda tener la aplicación de la medida y será según estos valores que cambiará la focalización del programa.

Los parámetros propuestos para Chile fueron definidos en base a aquellos utilizados en Estados Unidos en $1996^{23}$, ajustados por la razón entre el PIB per cápita de ese país en ese año y el PIB per cápita de Chile en 2009, cercana a 3. La Tabla $\mathrm{N}^{\circ} 1$ muestra los parámetros utilizados en la simulación.

La estructura creciente y luego decreciente del esquema tributario propuesto debe ser considerada, ya que si se cubre un tramo muy reducido de la distribución, es posible que se genere una resistencia a incrementar los ingresos para no perder el beneficio. En el extremo, a las personas no les convendría salir de la pobreza para recibir el crédito. Por lo anterior, se definió en esta propuesta que el subsidio se entregue a familias de los primeros seis deciles de ingreso.

Otra característica particular de esta propuesta es que los beneficios difieren según el tipo de familia beneficiaria. El máximo ingreso después de impuesto, es decir, el límite superior del tramo phase out para quienes no tienen hijos, es en promedio $\$ 147.675$ mensuales, y

${ }^{23}$ Las Tablas N ${ }^{\text {os. }}$ A4 y A5 del Anexo muestran los parámetros del EITC de 1996 y 2009 en Estados Unidos. 
TABLA $N^{\circ}$ 1: TRAMOS DE INGRESO, TASAS Y MONTOS DE CRÉDITO PROPUESTOS PARA CHILE

\begin{tabular}{ccc}
\hline Ingreso anual del hogar $(\mathrm{x})$ & Segmento & Crédito $(2$ hijos $)$ \\
$\$ 0-\$ 1.658 .311$ & Primer segmento & $40 \% * \mathrm{x}$ \\
$\$ 1.658 .311-\$ 2.165 .691$ & Fase plana & $\$ 663.324$ \\
$\$ 2.165 .691-\$ 5.315 .379$ & Tercer segmento & $\$ 663.324-21,06 \% *(\mathrm{x}-\$ 2.165 .691)$ \\
& & \\
Ingreso anual del hogar $(\mathrm{x})$ & Segmento & Crédito $(1$ hijo $)$ \\
$\$ 0-\$ 1.180 .777$ & Primer segmento & $34 \% * \mathrm{x}$ \\
$\$ 1.180 .777-\$ 2.165 .691$ & Fase plana & $\$ 401.464$ \\
$\$ 2.165 .691-\$ 4.677 .982$ & Tercer segmento & $\$ 401.464-15,98 \% *(\mathrm{x}-\$ 2.165 .691)$ \\
& & \\
Ingreso anual del hogar $(\mathrm{x})$ & Segmento & Crédito $(\mathrm{sin}$ hijos $)$ \\
$\$ 0-\$ 787.185$ & Primer segmento & $7,65 \% * \mathrm{x}$ \\
$\$ 787.185-\$ 984.914$ & Fase plana & $\$ 60.220$ \\
$\$ 984.914-\$ 1.772 .098$ & Tercer segmento & $\$ 60.220-7,65 \% *(\mathrm{x}-\$ 5.280)$ \\
& & \\
\hline
\end{tabular}

Fuente: Elaboración propia.

\$389.832 y \$442.948 según sean familias con uno y dos o más hijos respectivamente.

De lo anterior, una madre soltera con dos hijos y un sueldo mensual entre $\$ 138.193$ y $\$ 180.474$ (el salario mínimo en 2009 era $\$ 165.000)$, recibiría en la devolución de impuestos una transferencia monetaria por el monto máximo de subsidio, que son $\$ 663.324$ pesos anuales, equivalente a $\$ 55.277$ mensuales. Esto implicaría que esta familia tenga un ingreso per cápita entre $\$ 64.490$ y $\$ 78.583$, situándola sobre la línea de pobreza en 2009 (\$64.134).

\section{Resultados}

Debido a los distintos supuestos sobre la cantidad de horas que trabajan quienes se insertan al mercado laboral (20 o 45 horas) y los valores de la elasticidad de participación $(0 ; 0,1 ; 0,2 ; 0,3)$, se realizan siete simulaciones que combinan estos supuestos de la forma en que se presentan en la Tabla $\mathrm{N}^{\circ}$ 2. El escenario base (escenario A) es aquel en 
TABLA N ${ }^{\circ}$ 2: $\quad$ ESCENARIOS DE ANÁLISIS

Escenario Supuestos (horas/elasticidad de participación)

$\begin{array}{lc}\text { Escenario A } & 0 \text { horas } / \gamma_{\mathrm{k}}=0 \\ \text { Escenario B } & 20 \text { horas } / \gamma_{\mathrm{k}}=0,3 \\ \text { Escenario C } & 45 \text { horas } / \gamma_{\mathrm{k}}=0,3 \\ \text { Escenario D } & 20 \text { horas } / \gamma_{\mathrm{k}}=0,2 \\ \text { Escenario E } & 45 \text { horas } / \gamma_{\mathrm{k}}=0,2 \\ \text { Escenario F } & 20 \text { horas } / \gamma_{\mathrm{k}}=0,1 \\ \text { Escenario G } & 45 \text { horas } / \gamma_{\mathrm{k}}=0,1\end{array}$

Fuente: Elaboración propia en base a datos de Encuesta Casen 2009.

que la elasticidad es cero. Esto implica que las personas no modifican su comportamiento frente a cambios en el ingreso del trabajo, por lo que sólo acceden al beneficio quienes se encuentran trabajando inicialmente. Los casos restantes reconocen que se produce inserción laboral de quienes no están participando en el mercado del trabajo.

Los resultados son relativamente insensibles a cambios en las elasticidades de participación. Por esta razón y con el fin de simplificar la exposición de los resultados, se opta por focalizar el análisis primordialmente en tres escenarios. El escenario base (A), en el que la participación laboral permanece constante, es un buen punto de comparación ya que se aproxima al efecto mínimo esperado de la aplicación del EITC. Los escenarios B y C, correspondientes a una inserción laboral de 20 y 45 horas respectivamente, asumen una elasticidad de participación de 0,3 y que todas las mujeres que buscan empleo lo encuentran, representando el efecto máximo entre los distintos valores supuestos ${ }^{24}$.

\section{Focalización}

En el escenario base, que otorga crédito tributario sólo a quienes trabajan inicialmente, el programa beneficia potencialmente a 370.249 familias. Al considerar la inserción laboral en los escenarios siguientes se incrementa la cantidad de beneficiarios. Si la elasticidad es 0,3 y las mujeres entran a trabajar media jornada (escenario B), las familias que reciben el EITC aumentan a 510.886, mientras que si trabajan jornada

${ }^{24}$ Todas las estimaciones para el resto de los escenarios pueden ser solicitadas directamente a los autores. 
completa (escenario C) aumentan a 456.489 los núcleos beneficiados. La mayor participación observada cuando las mujeres trabajan media jornada se explica por el menor nivel de ingreso mensual que obtienen al trabajar menos horas, con lo cual acceden personas que en caso de trabajar 45 horas no serían elegibles.

Las Tablas Nos. 3 y 4 muestran la distribución por tipo de beneficiario, tanto directo como indirecto. Los beneficiarios directos son mujeres que trabajan y reciben el EITC. Los beneficiarios indirectos son hombres y mujeres que no reciben el EITC pero son parte del mismo núcleo que el de los beneficiarios directos. Más del 90\% de los beneficiarios directos son jefas de hogar y entre los beneficiarios indirectos predominan los menores de 18 años. La mínima cobertura ocurre en el escenario base con 371.139 beneficiarios directos y la máxima en el escenario B con $457.502^{25}$.

A partir de la Tabla $\mathrm{N}^{\circ} 5$ se comparan beneficiarias con no beneficiarias en base a algunas de sus características sociodemográficas como edad, educación, número de hijos e ingreso. Las beneficiarias son mujeres con bajos niveles de educación, salario por hora e ingreso per cápita, principalmente son solteras o separadas, tienen menor apoyo económico de otros integrantes del hogar, y más edad y más hijos que las no beneficiarias. Por otro lado, dos tercios de las no beneficiarias son casadas, provienen de familias con mayor nivel de ingreso y el $85 \%$ vive en hogares donde otros adultos trabajan. Entre las mujeres de los primeros seis deciles de ingreso, las no beneficiarias tienen mayor salario por hora, menos hijos elegibles, son casadas, y con mayor frecuencia viven en zonas rurales, fuera de la Región Metropolitana, y tienen alguna discapacidad.

En la Tabla $\mathrm{N}^{\circ} 6$ se muestra la distribución de beneficiarias y el monto de beneficio recibido por decil de ingreso. La inserción laboral mejora sustancialmente la focalización del programa, sobre todo cuando las mujeres entran a trabajar jornada completa, ya que estas últimas provienen de familias de menores ingresos, tienen más hijos elegibles, pero menor nivel educativo y menores salarios horarios. La inserción laboral además contribuye a homogeneizar la distribución de beneficiarias y beneficios por decil de ingreso, siendo las beneficiarias del segundo decil las que más se benefician del programa.

${ }^{25}$ El número de beneficiarios directos puede exceder al de las familias beneficiarias por la existencia del segundo tipo de beneficiarias descrito anteriormente, que permite que haya más de un receptor del EITC por familia. 


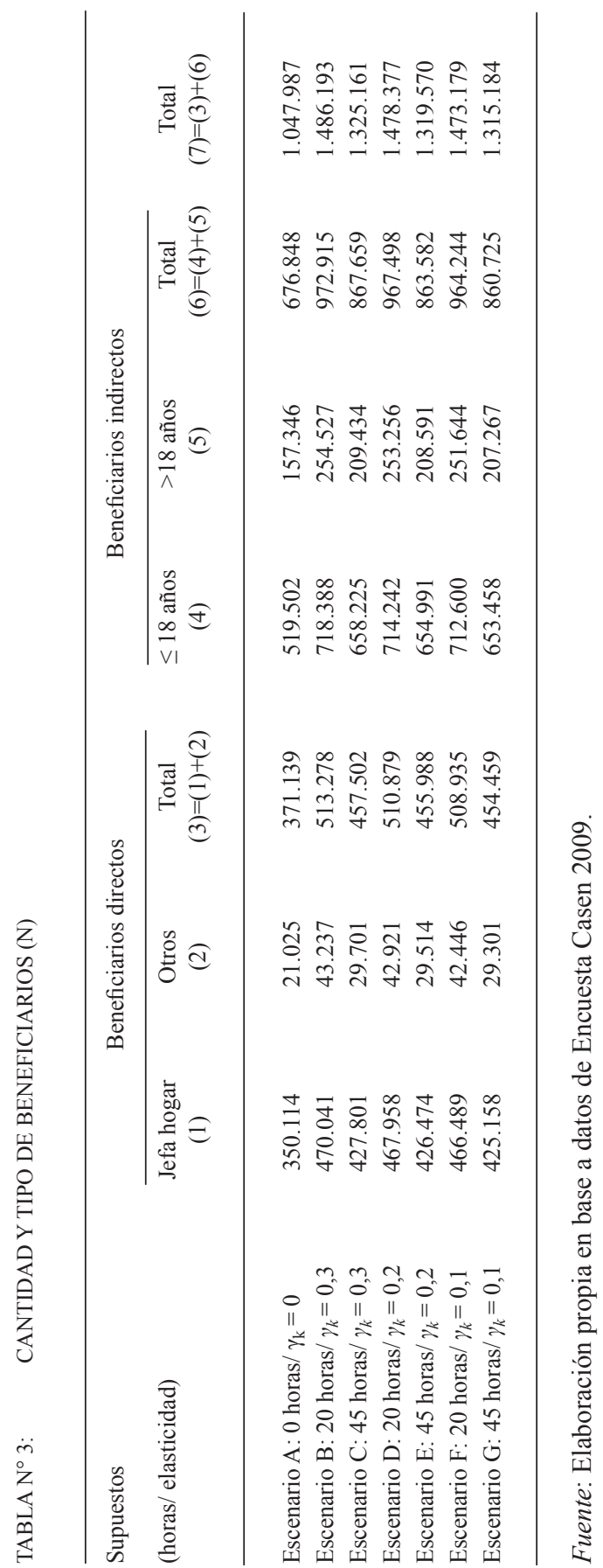




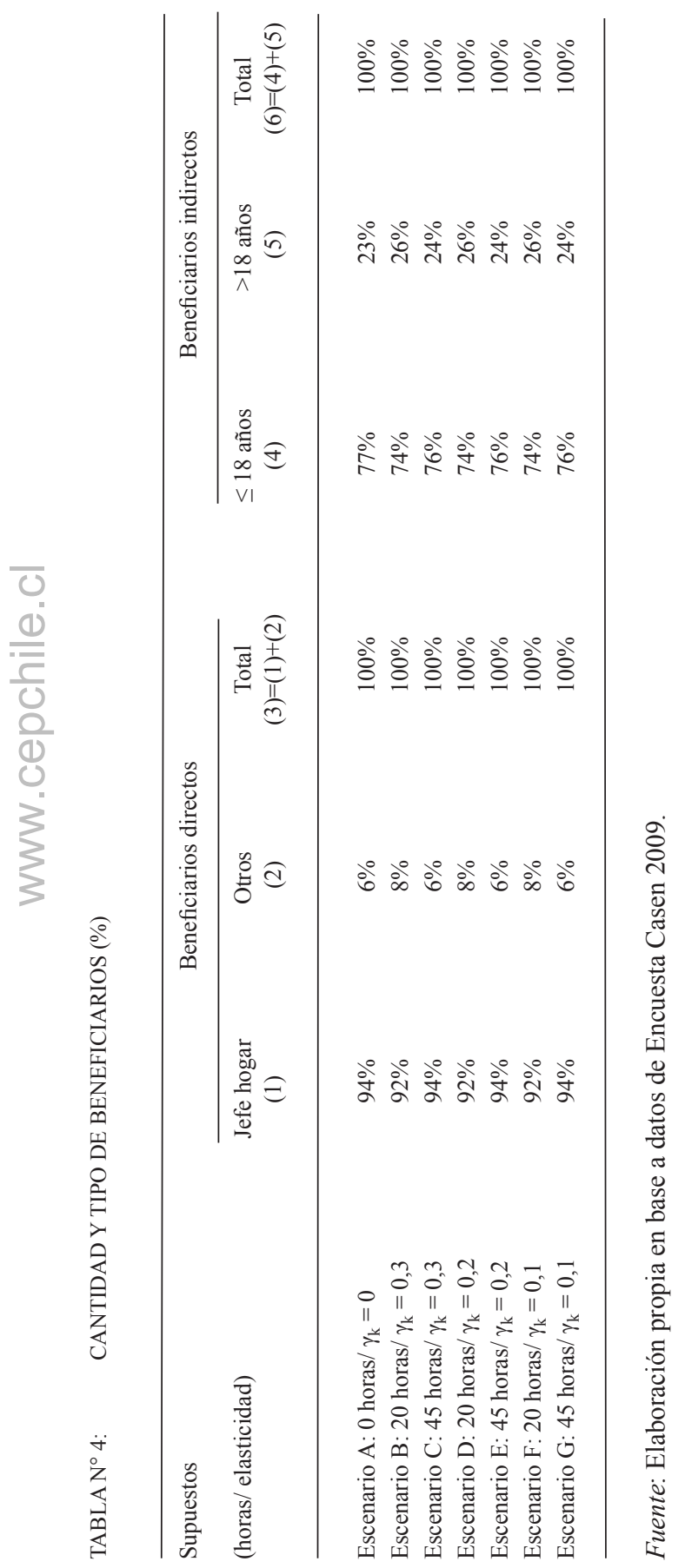




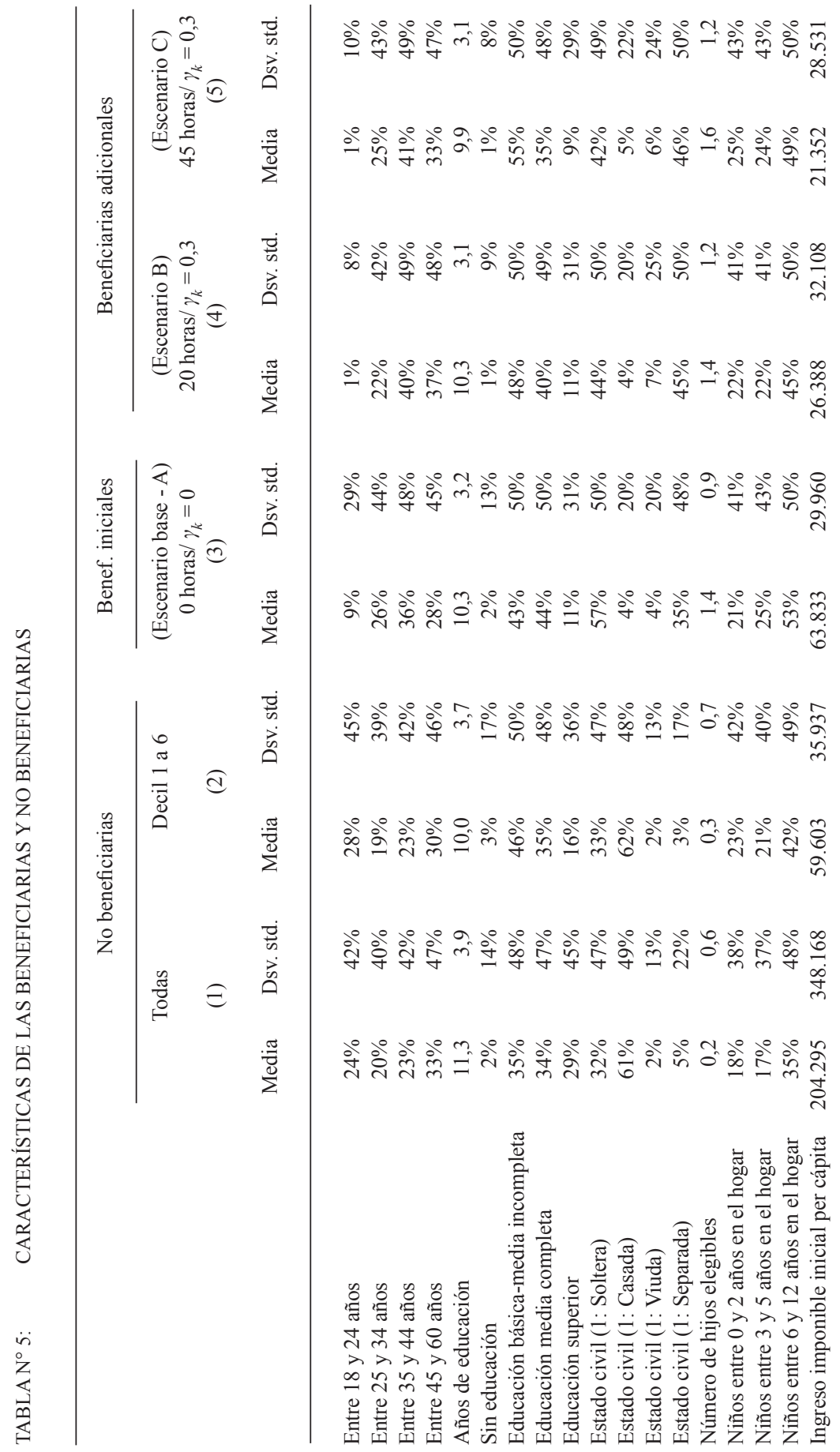




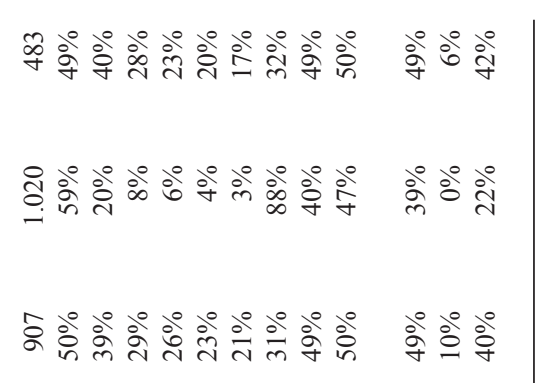

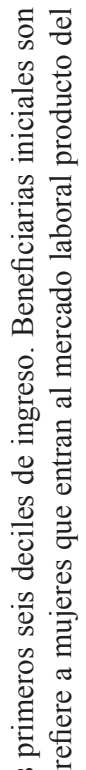

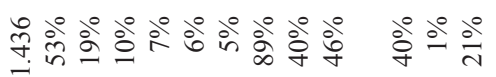

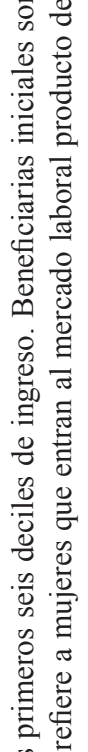

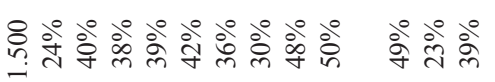

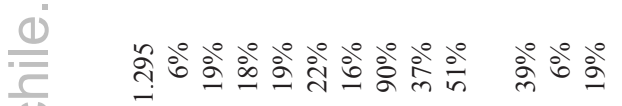

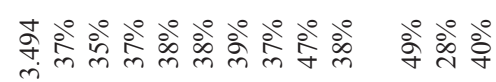

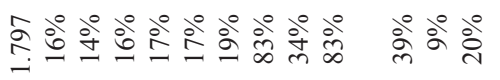

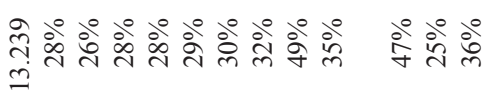

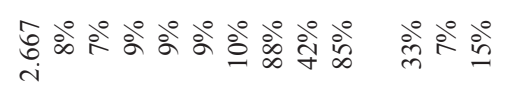

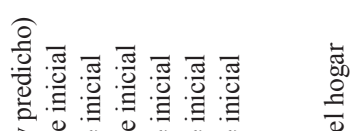

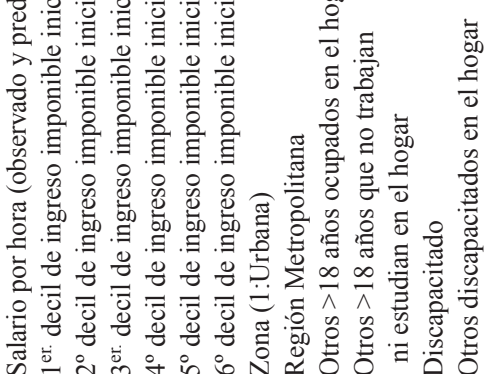

\&.

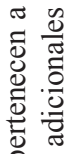

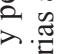

की

至造

$\approx$

ปै

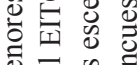

링ㅎํ 되

की है च

总 :-

$\infty$ 응 $\frac{\pi}{0}$

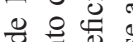

\% ह

흥

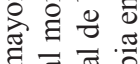

灵

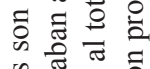

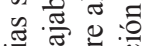

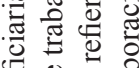

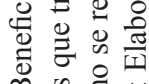
क के

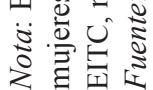


TABLA $N^{\circ} 6$ :

DISTRIBUCIÓN \% DE BENEFICIARIAS (N) Y BENEFICIOS (\$) POR DECIL DE INGRESO

\begin{tabular}{|c|c|c|c|c|c|c|}
\hline \multirow{3}{*}{ Decil de ingreso } & \multicolumn{2}{|c|}{ (Escenario A) } & \multicolumn{2}{|c|}{ (Escenario B) } & \multicolumn{2}{|c|}{ (Escenario C) } \\
\hline & \multicolumn{2}{|c|}{0 horas $/ \gamma_{k}=0$} & \multicolumn{2}{|c|}{20 horas $/ \gamma_{k}=0,3$} & \multicolumn{2}{|c|}{45 horas $/ \gamma_{k}=0,3$} \\
\hline & $(\mathrm{N})$ & $(\$)$ & $(\mathrm{N})$ & (\$) & $(\mathrm{N})$ & (\$) \\
\hline 1 & $6 \%$ & $4,2 \%$ & $19 \%$ & $17,3 \%$ & $16 \%$ & $14,8 \%$ \\
\hline 2 & $19 \%$ & $21,6 \%$ & $19 \%$ & $21,5 \%$ & $19 \%$ & $21,1 \%$ \\
\hline 3 & $18 \%$ & $21,1 \%$ & $15 \%$ & $17,9 \%$ & $16 \%$ & $18,6 \%$ \\
\hline 4 & $19 \%$ & $21,1 \%$ & $16 \%$ & $17,5 \%$ & $16 \%$ & $18,2 \%$ \\
\hline 5 & $22 \%$ & $21,0 \%$ & $18 \%$ & $16,8 \%$ & $19 \%$ & $17,7 \%$ \\
\hline 6 & $16 \%$ & $11,1 \%$ & $13 \%$ & $9,0 \%$ & $13 \%$ & $9,5 \%$ \\
\hline
\end{tabular}

Fuente: Elaboración propia en base a datos de Encuesta Casen 2009.

Un análisis equivalente se puede realizar respecto al número de hijos. La Tabla $\mathrm{N}^{\circ} 7$ muestra que el $85 \%$ de las beneficiarias tiene al menos un hijo elegible. El beneficio promedio en el escenario base es $\$ 360.453$ anuales, equivalente a $\$ 30.038$ mensuales. Una madre soltera con dos o más hijos recibe $45 \%$ más que una con un hijo, quien a su vez recibe ocho veces lo que obtiene una mujer sin hijos. La inserción laboral de mujeres más vulnerables se refleja en la caída del monto promedio de la transferencia en los escenarios B y C.

Finalmente, desde el punto de vista de la focalización, es importante considerar la distribución de beneficiarias y monto de beneficios por segmento del EITC (ver Tabla $\mathrm{N}^{\circ}$ 8). Las simulaciones muestran que la cantidad de recursos y beneficiarias es creciente en los tramos de beneficio, concentrándose ambos en el tercer segmento. El primer tramo y la fase plana cubren una proporción similar de beneficiarios aunque en esta última es superior la cantidad de recursos requeridos, ya que es aquí donde se otorga el máximo de EITC. Las mujeres con dos o más hijos utilizan más de la mitad del presupuesto, y las beneficiarias sin hijos representan el $1 \%$ de los recursos y se encuentran en su mayoría en el tercer tramo. 

$\begin{array}{ll}\text { TABLA N }{ }^{\circ} \text { : } & \text { DISTRIBUCIÓN DE BENEFICIARIOS (N) Y BENEFICIOS (\$) } \\ & \text { POR NÚMERO DE HIJOS }\end{array}$

\begin{tabular}{|c|c|c|c|c|}
\hline \multirow{2}{*}{ Supuestos (horas/elasticidad) } & \multicolumn{4}{|c|}{ Número de hijos elegibles } \\
\hline & Ninguno & Uno & Dos o más & Total \\
\hline \multicolumn{5}{|l|}{ Escenario A: 0 horas $/ \gamma_{k}=0$} \\
\hline Beneficiarios (N) & 50.818 & 170.378 & 149.943 & 371.139 \\
\hline Beneficiarios (\%) & $14 \%$ & $46 \%$ & $40 \%$ & $100 \%$ \\
\hline EITC Promedio (\$) & 37.680 & 340.655 & 492.343 & 360.453 \\
\hline Desv. std. (\$) & 18.117 & 80.606 & 164.634 & 188.284 \\
\hline Mín. & 314 & 3.497 & 2.396 & 314 \\
\hline Máx. & 60.220 & 401.464 & 663.324 & 663.324 \\
\hline \multicolumn{5}{|l|}{ Escenario B: 20 horas $/ \gamma_{k}=0,3$} \\
\hline Beneficiarios $(\mathrm{N})$ & 86.932 & 218.584 & 207.762 & 513.278 \\
\hline Beneficiarios (\%) & $17 \%$ & $43 \%$ & $40 \%$ & $100 \%$ \\
\hline EITC Promedio (\$) & 37.951 & 332.946 & 480.465 & 342.695 \\
\hline Desv. std. (\$) & 18.004 & 87.868 & 165.753 & 194.720 \\
\hline Mín. & 3.497 & 3.497 & 2.396 & 314 \\
\hline Máx. & 60.220 & 401.464 & 663.324 & 663.324 \\
\hline \multicolumn{5}{|l|}{ Escenario $\mathrm{C}: 45$ horas $/ \gamma_{k}=0,3$} \\
\hline Beneficiarios $(\mathrm{N})$ & 62.786 & 203.422 & 191.294 & 457.502 \\
\hline Beneficiarios (\%) & $14 \%$ & $44 \%$ & $42 \%$ & $100 \%$ \\
\hline EITC Promedio (\$) & 36.464 & 330.858 & 479.598 & 352.649 \\
\hline Desv. std. (\$) & 18.305 & 90.917 & 171.397 & 191.504 \\
\hline Mín. & 224 & 3.387 & 1.267 & 224 \\
\hline Máx. & 60.220 & 401.464 & 663.324 & 663.324 \\
\hline
\end{tabular}

Fuente: Elaboración propia en base a datos de Encuesta Casen 2009.

\section{Participación laboral}

El principal efecto esperado del EITC es aumentar la participación laboral. Conforme con esto, las simulaciones provocan efectivamente un desplazamiento a la derecha de la distribución de probabilidad de participación de quienes no trabajan inicialmente (ver Gráfico $\mathrm{N}^{\circ} \mathrm{A} 2$ del Anexo). Después de implementado el EITC, dependiendo de los supuestos de elasticidad y horas, la probabilidad aumenta entre 0,4 y 1,2 puntos porcentuales, lo que se traduce en la incorporación al mercado laboral de 142.139 y 86.363 mujeres en jornada parcial o completa respectivamente. Esto representa el 5,4\% y $3,3 \%$ del total de mujeres entre 18 y 60 años que no trabajan inicial- 
TABLA N ${ }^{\circ}$ :

DISTRIBUCIÓN \% DE BENEFICIARIAS (N) Y BENEFICIOS (\$) POR SEGMENTO DE EITC

\begin{tabular}{|c|c|c|c|c|}
\hline & Phase in & Fase plana & Phase out & Total \\
\hline Supuestos (horas/elasticidad) & $(\mathrm{N}) \quad(\$)$ & $(\mathrm{N}) \quad(\$)$ & $(\mathrm{N}) \quad(\$)$ & $(\mathrm{N})$ \\
\hline
\end{tabular}

Escenario A: 0 horas $/ \gamma_{k}=0$

\begin{tabular}{|c|c|c|c|c|c|c|c|c|c|}
\hline \multirow[t]{4}{*}{$\mathrm{N}^{o}$ hijos } & Ninguno & $3 \%$ & $0,3 \%$ & $2 \%$ & $0,3 \%$ & $9 \%$ & $1 \%$ & $14 \%$ & $1 \%$ \\
\hline & Uno & $7 \%$ & $5 \%$ & $16 \%$ & $18 \%$ & $22 \%$ & $20 \%$ & $46 \%$ & $43 \%$ \\
\hline & Dos o más & $9 \%$ & $12 \%$ & $7 \%$ & $13 \%$ & $24 \%$ & $30 \%$ & $40 \%$ & $55 \%$ \\
\hline & Total & $20 \%$ & $18 \%$ & $25 \%$ & $32 \%$ & $55 \%$ & $50 \%$ & $100 \%$ & $100 \%$ \\
\hline
\end{tabular}

Escenario B: 20 horas $/ \gamma_{k}=0,3$

\begin{tabular}{|c|c|c|c|c|c|c|c|c|c|}
\hline \multirow[t]{4}{*}{$\mathrm{N}^{o}$ hijos } & Ninguno & $5 \%$ & $1 \%$ & $2 \%$ & $0,4 \%$ & $10 \%$ & $1 \%$ & $17 \%$ & $2 \%$ \\
\hline & Uno & $8 \%$ & $7 \%$ & $15 \%$ & $18 \%$ & $19 \%$ & $17 \%$ & $43 \%$ & $41 \%$ \\
\hline & Dos o más & $12 \%$ & $15 \%$ & $7 \%$ & $13 \%$ & $22 \%$ & $29 \%$ & $40 \%$ & $57 \%$ \\
\hline & Total & $25 \%$ & $23 \%$ & $24 \%$ & $31 \%$ & $51 \%$ & $47 \%$ & $100 \%$ & $100 \%$ \\
\hline
\end{tabular}

Escenario C: 45 horas $/ \gamma_{k}=0,3$

\begin{tabular}{|c|c|c|c|c|c|c|c|c|c|}
\hline \multirow[t]{4}{*}{$\mathrm{N}^{o}$ hijos } & Ninguno & $3 \%$ & $0,3 \%$ & $2 \%$ & $0,3 \%$ & $9 \%$ & $1 \%$ & $14 \%$ & $1 \%$ \\
\hline & Uno & $6 \%$ & $5 \%$ & $15 \%$ & $17 \%$ & $23 \%$ & $19 \%$ & $44 \%$ & $42 \%$ \\
\hline & Dos o más & $9 \%$ & $12 \%$ & $7 \%$ & $13 \%$ & $25 \%$ & $31 \%$ & $42 \%$ & $57 \%$ \\
\hline & Total & $19 \%$ & $17 \%$ & $24 \%$ & $31 \%$ & $57 \%$ & $52 \%$ & $100 \%$ & $100 \%$ \\
\hline
\end{tabular}

Fuente: Elaboración propia en base a datos de Encuesta Casen 2009.

mente, y el 7,8\% y 4,7\% si se consideran sólo los primeros seis deciles de ingreso.

El cambio en la probabilidad de participación y la consiguiente inserción laboral se reflejan en la tasa de empleo femenino. La Tabla $\mathrm{N}^{\circ} 9$ presenta la tasa de empleo antes y después del EITC para distintos grupos de mujeres de 18 a 60 años. Primero se presenta la situación agregada, posteriormente la de las mujeres en los primeros seis deciles, y por último a las elegibles dentro de los primeros seis deciles de ingreso (entre paréntesis se muestra la desviación estándar).

Los resultados indican que la tasa de empleo aumenta en todos los grupos, aunque el incremento más importante se produce entre las mujeres elegibles, cuya participación laboral aumenta casi 20 puntos porcentuales en el escenario B ( 20 horas) y 12 puntos porcentuales en el escenario C (45 horas). El efecto es mayor cuando las mujeres entran a trabajar media jornada, debido a la mayor cantidad de beneficiarias que hay en el escenario B. Es importante destacar que estas magnitudes son 
TABLA No 9: $\quad$ TASA DE EMPLEO FEMENINO

Antes de EITC Después de EITC Después de EITC

20 horas $/ \gamma_{k}=0,3 \quad 45$ horas $/ \gamma_{k}=0,3$

Mujeres 18 a 60 años

Mujeres 18 a 60 años en deciles 1 a 6

Mujeres elegibles

$\begin{array}{cc}45,4 \% & 48,4 \% \\ (49,8 \%) & (50,0 \%) \\ 33,3 \% & 38,5 \% \\ (47,1 \%) & (48,7 \%) \\ 51,7 \% & 71,5 \% \\ (50,0 \%) & (45,2 \%)\end{array}$

\section{$45,4 \%$}

$33,3 \%$

$(47,1 \%)$

$(50,0 \%)$

Fuente: Elaboración propia en base a datos de Encuesta Casen 2009.

relevantes desde el punto de vista de su impacto en la posibilidad de generar ingresos autónomos permanentes que permitan a muchas familias salir de la pobreza.

\section{Pobreza}

Los efectos del EITC sobre la pobreza son medidos a través de tres indicadores: (i) la tasa de pobreza, que mide la proporción del total de la población que está bajo la línea de la pobreza (LP); (ii) la brecha de pobreza, que indica la diferencia entre el ingreso y la LP de quienes son considerados pobres; y (iii) la brecha de pobreza al cuadrado, que introduce una medida de la distribución del ingreso dentro del grupo de personas pobres, dando mayor peso a quienes tienen mayores brechas de pobreza (Foster, Greer y Thorbecke 1984).

Las Tablas $N^{\text {os. }} 10$ y 11 reportan respectivamente los resultados para el total de la población y para las familias donde la jefa de hogar es madre soltera. La primera fila describe el nivel inicial del índice y las siguientes el cambio ocurrido en cada escenario analizado, calculado como la diferencia entre el valor estimado después del EITC y el valor previo a la implementación del crédito. Los cálculos se basan en el ingreso per cápita de la familia después de impuesto y es por esto que las cifras no coinciden con las cifras oficiales de pobreza del año 2009.

La tasa de pobreza agregada se reduce entre 1 y 2 puntos porcentuales y junto a ello disminuye también la severidad de la pobreza y la cantidad de recursos necesarios para erradicarla. Una vez más la 
TABLA N ${ }^{\circ}$ 10: $\quad$ ÍNDICES DE POBREZA ANTES Y DESPUÉS DEL EITC:

TOTAL DE LA POBLACIÓN

\begin{tabular}{lccc}
\hline & $\begin{array}{c}\text { Tasa de } \\
\text { pobreza }\end{array}$ & $\begin{array}{c}\text { Brecha de } \\
\text { pobreza }\end{array}$ & $\begin{array}{c}\text { Brecha de } \\
\text { pobreza }\end{array}$ \\
\hline Antes de EITC & 0,225 & 0,101 & 0,069 \\
Cambio después de EITC & $(0,004)$ & $(0,002)$ & $(0,002)$ \\
Escenario A: 0 horas $/ \gamma_{k}=0$ & $-0,010^{* * *}$ & $-0,004^{* * *}$ & $-0,002^{* * * *}$ \\
& $(0,001)$ & $(0,000)$ & $(0,000)$ \\
Escenario B: 20 horas $/ \gamma_{k}=0,3$ & $-0,022^{* * *}$ & $-0,014^{* * *}$ & $-0,011^{* * *}$ \\
& $(0,001)$ & $(0,001)$ & $(0,000)$ \\
Escenario C: 45 horas $/ \gamma_{k}=0,3$ & $-0,021^{* * *}$ & $-0,012^{* * * *}$ & $-0,009^{* * *}$ \\
& $(0,001)$ & $(0,001)$ & $(0,000)$ \\
\hline
\end{tabular}

Nota: Error estándar calculado con el método de bootstrap en paréntesis. ${ }^{*} p<0,10$, $* * p<0,05, * * * p<0,01$.

Fuente: Elaboración propia en base a datos de Encuesta Casen 2009.

TABLA N ${ }^{\circ} 11: \quad$ ÍNDICES DE POBREZA ANTES Y DESPUÉS DEL EITC:

FAMILIAS CON JEFA DE HOGAR MADRE SOLTERA

\begin{tabular}{lccc}
\hline & $\begin{array}{c}\text { Tasa de } \\
\text { pobreza }\end{array}$ & $\begin{array}{c}\text { Brecha de } \\
\text { pobreza }\end{array}$ & $\begin{array}{c}\text { Brecha de } \\
\text { pobreza }^{\wedge 2}\end{array}$ \\
\hline Antes de EITC & 0,402 & 0,224 & 0,169 \\
Cambio después de EITC & $(0,007)$ & $(0,005)$ & $(0,004)$ \\
Escenario A: 0 horas $/ \gamma_{k}=0$ & $-0,049^{* * *}$ & $-0,018^{* * *}$ & $-0,010^{* * *}$ \\
Escenario B: 20 horas $/ \gamma_{k}=0,3$ & $(0,003)$ & $(0,001)$ & $(0,001)$ \\
& $-0,110^{* * *}$ & $-0,070^{* * *}$ & $-0,054^{* * *}$ \\
Escenario C: 45 horas $/ \gamma_{k}=0,3$ & $(0,005)$ & $(0,003)$ & $(0,002)$ \\
& $-0,107^{* * *}$ & $-0,061^{* * *}$ & $-0,045^{* * *}$ \\
& $(0,005)$ & $(0,003)$ & $(0,002)$ \\
\hline
\end{tabular}

Nota: Error estándar calculado con el método de bootstrap en paréntesis. ${ }^{*} p<0,10$, $* * p<0,05, * * * p<0,01$.

Fuente: Elaboración propia en base a datos de Encuesta Casen 2009. 
inserción laboral mejora significativamente los resultados agregados, independientemente de si las mujeres trabajen 20 o 45 horas, bajando la pobreza en más del doble respecto al escenario en que se asume que no hay efectos en la participación laboral.

Los resultados son bastante mayores en el caso de las familias con jefas madres solteras: la pobreza cae entre 5 y 11 puntos porcentuales y si inicialmente se requería $22 \%$ de ingresos adicionales para salir de la pobreza, con el EITC este requerimiento cae a $16 \%$.

La Tabla $\mathrm{N}^{\circ} 12$ presenta los valores calculados de los índices de pobreza para quienes participan en el EITC. Para cada caso analizado se presenta la situación inicial y el cambio en los índices de pobreza, expresado como la diferencia entre la situación posterior y previa al EITC. En línea con los resultados de focalización, las beneficiarias en los escenarios $\mathrm{B}$ y $\mathrm{C}$ provienen de familias más vulnerables que lo observado en el escenario base. Sin embargo, después de aplicar el EITC se reducen estas diferencias y se alcanza una situación final más homogénea entre los tres escenarios analizados, con una tasa de pobreza en torno a $23 \%$, una brecha de pobreza de 0,07 y una brecha de pobreza al cuadrado de

TABLA N ${ }^{\circ}$ 12: $\quad$ ÍNDICES DE POBREZA ANTES Y DESPUÉS DE EITC: GRUPO DE BENEFICIARIAS

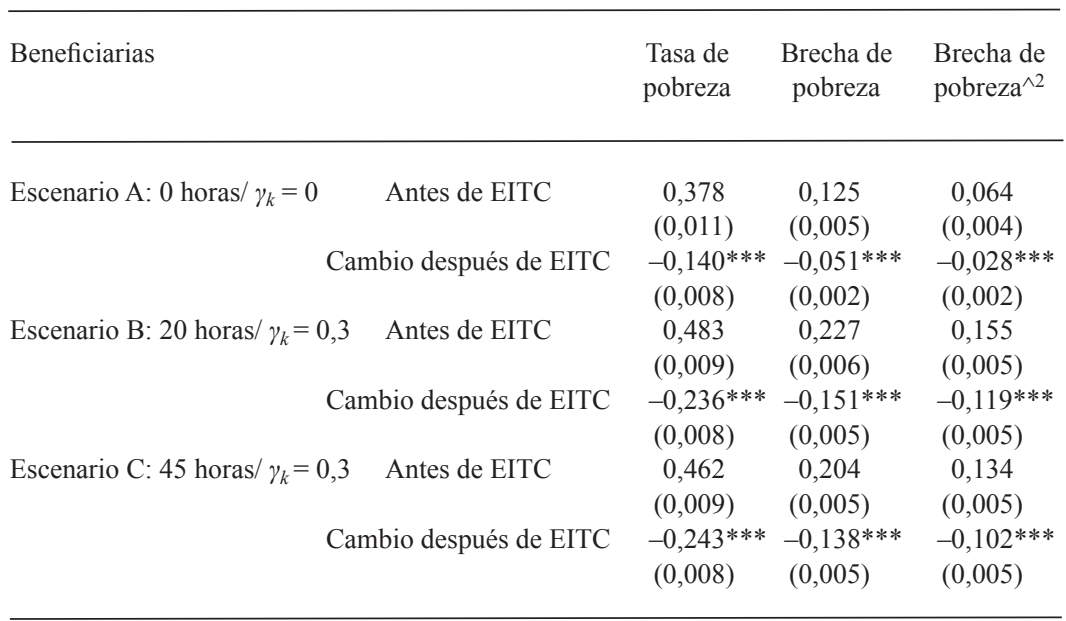

Nota: Error estándar calculado con el método de bootstrap en paréntesis. ${ }^{*} p<0,10$, $* * p<0,05, * * * p<0,01$.

Fuente: Elaboración propia en base a datos de Encuesta Casen 2009. 
0,035. En resumen, la evidencia de estas simulaciones indica que existe movilidad de ingresos después de aplicar el EITC y que la inserción laboral genera cambios de mayor magnitud en los índices de pobreza con respecto a la situación base.

\section{Desigualdad}

El EITC implementado en estas simulaciones lo reciben personas de los primeros seis deciles de ingreso, por lo que se esperaría que tuviese un efecto en reducir las brechas existentes entre el ingreso de pobres y ricos, mejorando así la distribución del ingreso. La Tabla $\mathrm{N}^{\circ} 13$ presenta el nivel inicial y el cambio en los indicadores de desigualdad, calculado como la diferencia entre el valor estimado después del EITC y el valor previo a la implementación del crédito. Se observa una reducción significativa de alrededor de 0,01 puntos en el coeficiente de Gini, que inicialmente es de 0,51 . Las brechas de ingreso entre distintos percentiles de la población también se reducen, mejorando la posición relativa de los más pobres en la distribución del ingreso.

A partir del cambio que se produce en la fracción del ingreso total que recibe cada decil de la población después de aplicar el EITC, se desprende que las familias pobres obtienen una mayor proporción del ingreso total, la que por otro lado se reduce para el $40 \%$ más rico de la

\begin{tabular}{lclll}
\hline & $\mathrm{p} 10 / \mathrm{p} 50$ & $\mathrm{p} 75 / \mathrm{p} 25$ & $\mathrm{p} 90 / \mathrm{p} 10$ & Gini \\
\hline & & & & \\
Antes de EITC & 0,357 & 2,987 & 9,264 & 0,512 \\
& $(0,004)$ & $(0,041)$ & $(0,293)$ & $(0,006)$ \\
Cambio después de EITC & & & & \\
Escenario A: 0 horas $/ \gamma_{k}=0$ & $0,007^{* * *}$ & $-0,077^{* * *}$ & $-0,237^{* * *}$ & $-0,003^{* * *}$ \\
& $(0,001)$ & $(0,006)$ & $(0,033)$ & $(0,000)$ \\
Escenario B: 20 horas $/ \gamma_{k}=0,3$ & $0,026^{* * *}$ & $-0,138^{* * *}$ & $-0,765^{* * *}$ & $-0,008^{* * *}$ \\
& $(0,002)$ & $(0,010)$ & $(0,056)$ & $(0,000)$ \\
Escenario C: 45 horas $/ \gamma_{k}=0,3$ & $0,022^{* * *}$ & $-0,141^{* * *}$ & $-0,695^{* * *}$ & $-0,008^{* * *}$ \\
& $(0,002)$ & $(0,010)$ & $(0,055)$ & $(0,000)$ \\
& & & & \\
\hline
\end{tabular}

Nota: Error estándar calculado con el método de bootstrap en paréntesis. ${ }^{*} p<0,10$, $* * p<0,05, * * * p<0,01$.

Fuente: Elaboración propia en base a datos de Encuesta Casen 2009. 
población, todo lo cual es consistente con la estructura del esquema de EITC aplicado. Además, los resultados indican que en el escenario base los principales favorecidos con el crédito tributario propuesto son los individuos de los deciles 3 y 4, mientras que si hay inserción laboral, los más beneficiados son los miembros de las familias pertenecientes a los primeros tres deciles de ingreso, reiterando el rol del efecto de la participación laboral en la focalización de los recursos.

Finalmente, se debe destacar que para evaluar la aplicación de un crédito tributario al ingreso hay que considerar también la política de financiamiento del programa (Mirrlees et al. 2010). Si bien un esquema tributario como el EITC es progresivo, para que los efectos en la distribución del ingreso se materialicen es necesario que la fuente de financiamiento del programa no sea regresiva - por ejemplo, a partir de un aumento en el IVA ${ }^{26}$ - ya que esto podría reducir los efectos positivos del programa en la equidad y empeorar la situación relativa de los más vulnerables. De igual forma, el financiamiento con IVA disminuye el ingreso disponible de los más pobres, con lo que el efecto neto en pobreza del EITC sería ambiguo. Esto no ocurre si se financia con un impuesto al ingreso, ya que los más pobres están exentos. Por lo tanto, entre las alternativas de financiamiento disponibles se debieran preferir impuestos progresivos, que incluso mejorarían aun más los indicadores de desigualdad, o la sustitución de programas sociales con resultados insuficientes o que sólo logran cambios de corto plazo sin afectar la capacidad permanente de generar mayores ingresos autónomos de las familias.

\section{Robustez de los resultados}

Uno de los supuestos clave del modelo utilizado en las simulaciones es que la elasticidad de oferta laboral es nula respecto a las horas trabajadas. Es relevante entonces sensibilizar las simulaciones respecto a este supuesto para determinar qué tan robustos son los resultados.

$\mathrm{Al}$ analizar un escenario alternativo (escenario $\mathrm{H}$ ) en el que las mujeres se insertan al mercado laboral para trabajar 45 horas, con una una elasticidad de participación de 0,3 y una elasticidad de horas de

${ }^{26}$ La evidencia en la literatura económica es robusta en mostrar que el IVA es un impuesto regresivo (Caspersen y Metcalf 1994, Decoster et al. 2010, Leahy et al. 2011), incluyendo Chile (Cantallops et al. 2007). 
$0,1^{27}$, las simulaciones muestran que el número total de beneficiarias no cambia ya que el nuevo ingreso no alcanza a sobrepasar el límite superior del tramo phase out. Sin embargo, se desplaza hacia la derecha la distribución de horas trabajadas, lo que aumenta las beneficiarias y los recursos destinados en el tercer tramo, principalmente por el traspaso de mujeres con hijos desde la fase plana. Todo esto se traduce también en una caída del costo del programa ( $0,2 \%$ inferior), ya que con el aumento de beneficiarias en el tramo phase out, donde el crédito es decreciente, cae el monto promedio de beneficios del EITC.

La variación en los efectos en pobreza y desigualdad, medida esta última como el cambio en la razón del ingreso promedio de distintos percentiles, es muy pequeña respecto al escenario $\mathrm{C}$ ya analizado ${ }^{28}$. No obstante, el coeficiente de Gini aumenta debido a que quienes están recibiendo más dinero son los menos pobres entre los beneficiarios (el detalle se encuentra en las Tablas $\mathrm{N}^{\text {os. }}$ A6 y A7 del Anexo). En un segundo año de aplicación, las horas trabajadas también se moverían para quienes se insertaron al mercado laboral con el EITC, por lo que se esperaría que baje aun más la desigualdad y mejore la focalización de los recursos.

Se analizó también la robustez de los resultados en el supuesto de que las mujeres con una probabilidad de participación laboral mayor que 0,5 sean las que se insertan en el mercado laboral. Para estos efectos se consideró la posibilidad de que ello ocurriera para las mujeres con una probabilidad de participación ex post igual o mayor que 0,6. Bajo esta regla, quienes deciden participar son mujeres relativamente más educadas, menos jóvenes, en mayor proporción separadas, con menos apoyo económico en el hogar y que provienen de familias más vulnerables aunque tienen un mayor salario por hora.

Los resultados indican que en este caso se reduce el número de beneficiarias y cambia la distribución por número de hijos y por tramo de beneficio, aumentando la proporción de mujeres con hijos y la cantidad de beneficiarias en el segundo y tercer segmento. La menor cantidad de beneficiarias provoca una caída del costo del programa de alrededor de $7 \%$, a pesar de que el monto promedio que recibe cada

${ }^{27}$ Se respeta el consenso teórico de que la elasticidad de oferta de horas es menor que la de participación.

${ }^{28}$ Con excepción del indicador p10/p50, todas las diferencias entre los escenarios $\mathrm{C}$ y $\mathrm{H}$ son estadísticamente distintas de cero. 
participante aumenta. Los efectos en participación, pobreza y desigualdad son similares a los de los escenarios B y C analizados, aunque de menor magnitud (ver Tablas $\mathrm{N}^{\text {os. }}$ A8 y A9 en el Anexo). Los escenarios analizados son llamados B-1 y C-1, debido a que son una variación de los casos B y C.

Por último, se utilizaron distintas especificaciones de la ecuación de selección del modelo de Heckman estimado y los resultados no experimentaron grandes variaciones.

\section{Comparación entre el EITC y el Programa Ingreso Ético Familiar (IEF)}

La propuesta de EITC para Chile que se simula en este trabajo fue motivada, entre otras cosas, por el lanzamiento en marzo de 2011 del Programa Asignación Social, considerado el primer paso hacia el Programa Ingreso Ético Familiar (IEF) que comenzará a regir a partir del año 2013. Este programa remplazará al actual Chile Solidario y pretende erradicar la pobreza extrema en el país en el 2014.

Los montos de las transferencias y requisitos para los beneficiarios del IEF serán establecidos en un reglamento comprometido para enero de 2013, por lo que no son conocidos al momento de escribir este trabajo. Sin embargo, la ley promulgada establece que los beneficiarios participarán en el programa por un mínimo de 12 y un máximo de 24 meses, y los beneficios consistirán en: una transferencia base, un subsidio por dignidad ${ }^{29}$, transferencias condicionadas a actividades de salud y educación, y bonos por logros. Estos últimos incluyen un bono por formalización y un bono por graduación de enseñanza media que se entregarán una sola vez; y un bono por esfuerzo que se entregará una vez al año a quienes pertenezcan al 30\% más vulnerable de la población que logren desempeños destacados o de superación en las áreas de educación, salud, empleo, ahorro y en rehabilitación de drogas.

El programa incluye además un subsidio al empleo de las mujeres entre 25 y 60 años que trabajen y pertenezcan al $40 \%$ más vulnerable de la población. Las inscripciones a este subsidio al salario comenzaron

${ }^{29}$ Incluye subsidios de consumo de agua potable, servicio de alcantarillado de aguas servidas, subvención educacional pro retención de alumnos y bono de protección. Todas estas prestaciones actualmente son parte del Programa Chile Solidario. 
en julio de 2012 y el monto que se entregue se distribuirá entre las beneficiarias $(20 \%)$ por un plazo de cuatro años y sus empleadores $(10 \%)$ por un total de 24 meses. El subsidio otorgará como máximo un monto mensual de \$34.079 equivalentes a \$408.948 al año.

El Programa de Asignación Social consiste en la entrega de un bono base mensual (incondicional) por individuo y cinco transferencias condicionadas a actividades de salud preventiva, educación, y un incentivo a la inserción laboral femenina, el que se materializa en un subsidio al salario para mujeres mayores de 18 años que no hayan trabajado durante los dos años previos a la inscripción, y que registren al menos tres cotizaciones continuas en los siguientes diez meses. Las beneficiarias de este último componente pasarán a ser beneficiarias del subsidio a la mujer cuando entre en vigencia el IEF. La Tabla $\mathrm{N}^{\circ} \mathrm{A} 10$ del Anexo detalla los montos y requisitos del programa.

Dados los recursos que ya se han gastado tanto en el Programa Chile Solidario original, como los costos asociados a la Asignación Social y al subsidio al empleo de la mujer, nos parece relevante, y además informativo, comparar la efectividad y los costos de ambos tipos de política pública. Para esto se utiliza información secundaria proveniente de una microsimulación realizada por Acero y Cabezas (2011) — de donde se obtienen los efectos en pobreza, desigualdad y empleo- - e información del presupuesto nacional (Dipres) del año 2012 para los datos de costos.

Acero y Cabezas (2011), a través de una microsimulación, evalúan, sobre el universo de beneficiarios del Programa Chile Solidario, el efecto que tendría la aplicación del Programa Asignación Social y un subsidio al empleo de hombres y mujeres ${ }^{30}$. Si los beneficiarios además, por ser parte del Chile Solidario, reciben los componentes del subsidio por dignidad, el programa simulado es muy similar al Ingreso Ético Familiar, aunque con mayor cobertura ya que los autores, además de incluir a hombres en la recepción del subsidio al empleo, consideran

30 Los autores simulan un subsidio basado en las recomendaciones del Consejo de Trabajo y Equidad, configurado en 2007, que consiste en un subsidio del $30 \%$ del salario, distribuido entre el empleador $(10 \%)$ y el beneficiario (20\%), con la misma estructura del subsidio al empleo joven y que alcanza el máximo en una renta mensual de $\$ 167.000$ y que decrece hasta llegar a cero para rentas superiores a $\$ 334.000$. Esto supone un bono máximo mensual de \$33.400. En la simulación del Programa Asignación Social no incluyen el bono por desempeño escolar, ya que no era parte de los componentes en 2011. 
como beneficiarios a la totalidad de las familias que declaren en la Encuesta Casen 2009 participar en el programa Chile Solidario (273.732 familias) y no sólo a las 170.000 que anunció oficialmente el gobierno para el Programa Asignación Social.

Los resultados de la microsimulación muestran que el IEF reduce el coeficiente de Gini en 0,001 puntos y la tasa de pobreza cae 0,6 puntos porcentuales (de 15,1\% a 14,5\%), aunque los autores no reportan el error estándar de sus estimaciones, por lo que no es posible saber si estas diferencias son estadísticamente significativas. No obstante, los efectos son inferiores a los obtenidos al simular un crédito tributario al ingreso, el cual reduce el coeficiente de Gini en 0,008 puntos y la pobreza en 2 puntos porcentuales. Además, mientras el EITC tiene la capacidad de disminuir la pobreza e incentivar el trabajo de forma simultánea, Acero y Cabezas (2011) muestran que el componente para fomentar la participación laboral se ve anulado por el aumento del ingreso no laboral, generando así un desincentivo al empleo, sobre todo en el grupo de mujeres y personas no jefas de hogar. Adicionalmente, se reduce el número de horas de trabajo agregadas, aunque entre quienes permanecen en el mercado del trabajo se observa un pequeño aumento.

Con los datos disponibles también es posible comparar el cambio porcentual en el ingreso per cápita promedio de cada decil después de la aplicación de ambos programas, lo cual se presenta en el Gráfico $\mathrm{N}^{\circ} 3$. En la comparación entre ambos, el EITC tiene una mejor focalización, sobre todo cuando hay inserción laboral. Con excepción del efecto en el primer decil en el escenario base, que corresponde al mínimo efecto posible, es claro que el EITC domina al IEF simulado en los primeros seis deciles de ingreso; en los escenarios donde ocurre inserción laboral, el efecto del EITC para los primeros dos deciles es más de siete veces lo observado para el IEF.

Es importante notar que el IEF simulado tiene una magnitud muy superior a lo establecido por el gobierno respecto al programa de Asignación Social, a pesar de lo cual su cobertura es inferior a la del crédito al ingreso propuesto en este trabajo. De hecho, mientras que el IEF fue concebido como un programa para combatir la extrema pobreza, el EITC tiene por objeto mejorar el bienestar de las familias y generar mayor participación laboral tanto en la población más vulnerable como en los estratos medios (quintil 5). Las diferencias en el tamaño de los grupos de beneficiarios y en el tipo de beneficiario (el EITC en promedio 
GRÁFICO N ${ }^{\circ}$ : CAMBIO EN INGRESO PER CÁPITA PROMEDIO POR DECIL

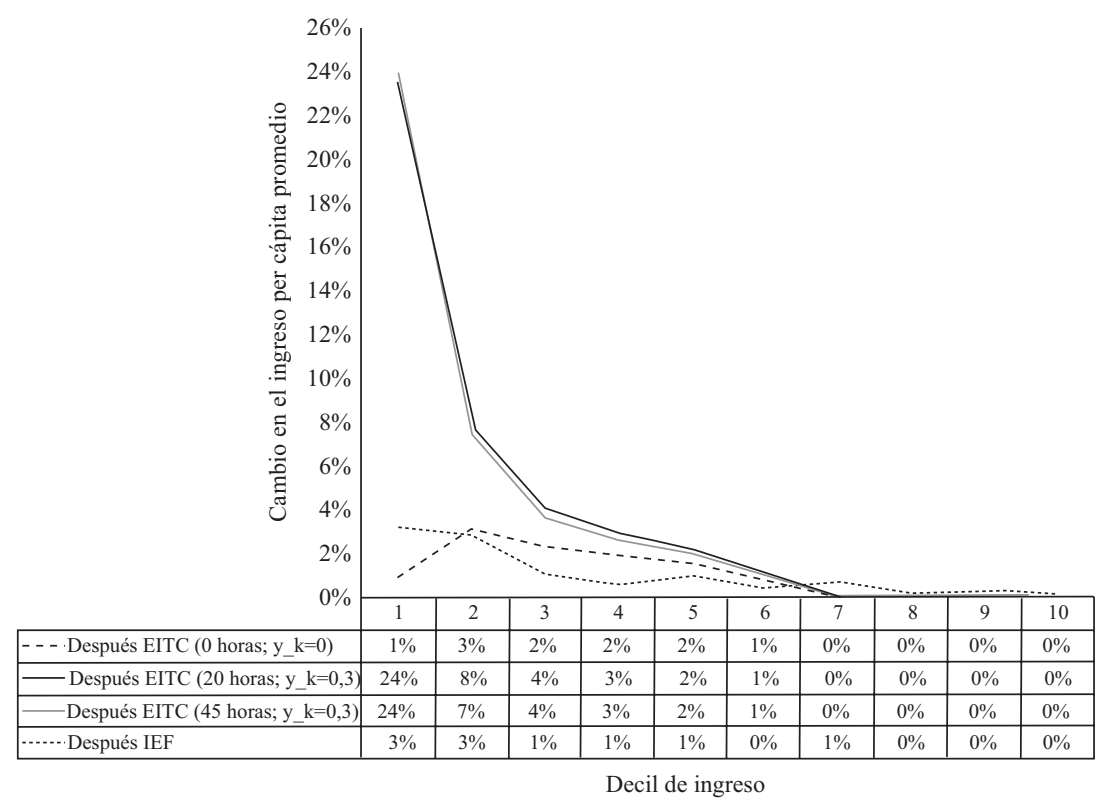

Fuentes: Elaboración propia en base a datos de Encuesta Casen 2009. Datos de IEF obtenidos de microsimulación realizada por Acero y Cabezas (2011).

tendría beneficiarios de mayores ingresos), dificultan la comparación entre ambas alternativas, ya que lo observado podría potencialmente ser un resultado relacionado al tamaño y tipo de beneficiario y no al tipo de política implementada.

Sin embargo, si restringimos los beneficios del crédito tributario a las 170.000 familias más pobres entre aquellas que recibían el EITC con la simulación, y se utilizan los supuestos del escenario $\mathrm{C}$, los resultados muestran que la caída en pobreza y desigualdad sigue siendo significativa y superior a lo encontrado por Acero y Cabezas (2011): la pobreza cae $1,5 \%$ y el coeficiente de Gini en 0,006 puntos. Además, con una focalización exclusiva en los primeros tres deciles de ingreso, este EITC restringido aumenta el ingreso entre los más vulnerables en casi cuatro veces más que el Ingreso Ético Familiar simulado.

Respecto a los costos de implementación, dependiendo de los supuestos utilizados, la aplicación del EITC en Chile significaría un 
gasto en transferencias mínimo de $\$ 143,1$ mil millones en el escenario base, el cual se incrementaría con la inserción laboral a \$188,2 mil millones en el escenario B y a $\$ 172,6$ mil millones en el escenario C. Por su parte, según datos de la Dirección de Presupuestos, el Programa Ingreso Ético Familiar tiene asignado para el año 2012 \$81,6 mil millones $^{31}$. Esto representa el $0,3 \%$ del presupuesto total nacional, mientras que el crédito tributario al ingreso propuesto en este trabajo requiere entre $0,5 \%$ y $0,6 \%$ de los recursos para el gasto en transferencias.

La diferencia observada en el costo de los programas también interfiere la interpretación de la comparación realizada en el Gráfico $\mathrm{N}^{\circ}$ 3. Por esta razón, simulamos los efectos que tendría un EITC si se dispusiera de la misma cantidad de recursos que para el IEF. Los resultados señalan consistentemente que con $\$ 81,6$ mil millones $(\$ 76,2$ mil millones de 2009) se reduciría significativamente la pobreza en 1,7\% y el coeficiente de Gini caería en 0,006 puntos. Además, focalizado en los primeros cuatro deciles de ingreso, este EITC restringido tiene asociados aumentos de ingreso de hasta cuatro veces lo observado con el Ingreso Ético Familiar entre los más vulnerables.

La Tabla $\mathrm{N}^{\circ} 14$ muestra el costo de cada programa, el número de familias beneficiarias y el costo por familia. Para el EITC se muestran los distintos escenarios analizados, para así tener un rango de costos asociados. Si bien el costo total del crédito propuesto duplica lo destinado al IEF, hay que considerar que el EITC no sólo triplica la cantidad de familias beneficiadas, lo que se traduce en un costo por familia $25 \%$ inferior al IEF, sino que también promueve la inserción laboral femenina y en el largo plazo debiera reducir la dependencia del sistema de bienestar social.

Estos resultados no son sorprendentes por cuanto son consistentes con una estructura de beneficios con fuertes incentivos, sobre todo en el caso de la mujeres, a abandonar el mercado laboral y recibir

${ }^{31}$ Con la información disponible este presupuesto representa potencialmente una cota mínima del costo del Programa Ingreso Ético Familiar, ya que los $\$ 81,6$ mil millones corresponden a la partida de Bonificación al Ingreso Familiar y el subsidio al empleo será financiado por el Servicio Nacional de Capacitación y Empleo. Sin embargo la ley del IEF establece que el mayor gasto fiscal que represente la aplicación del IEF durante el 2012 se financiará con presupuesto del Ministerio de Desarrollo Social y el Tesoro Público. 
TABLA No $14: \quad$ COSTOS ASOCIADOS A LOS PROGRAMAS IEF Y EITC

\begin{tabular}{lccc}
\hline Programa & Costo total (\$) & Familias beneficiarias & $\begin{array}{c}\text { Costo por familia } \\
\$\end{array}$ \\
\hline Ingreso Ético Familiar & 81.597 .178 .000 & 170.000 & 479.983 \\
Earned Income Tax Credit & & & \\
$\quad$ Escenario A: 0 horas $/ \gamma_{k}=0$ & 143.169 .545 .222 & 370.249 & 361.320 \\
Escenario B: 20 horas $/ \gamma_{k}=0,3$ & 188.246 .025 .627 & 510.886 & 344.300 \\
Escenario C: 45 horas $/ \gamma_{k}=0,3$ & 172.663 .264 .829 & 456.489 & 353.431 \\
& & & \\
\hline
\end{tabular}

Nota: Los montos están en pesos de diciembre de 2011, mes de elaboración del presupuesto nacional.

Fuente: Elaboración propia en base a datos de Encuesta Casen 2009 y Presupuesto Nacional 2012 (Dipres (2012)).

exclusivamente las transferencias condicionadas y la trasferencia base, no condicionada, que representa el $47 \%$ del beneficio del Programa Asignación Social ${ }^{32}$.

En relación con los costos, una característica del Programa Ingreso Ético Familiar que lo encarece respecto al EITC es que el subsidio es estrictamente creciente con el número de integrantes de la familia. Esto distorsiona aun más la decisión de participar en el mercado laboral de jefas de familias numerosas y no reconoce las economías de escala que hay dentro del hogar. Además, el EITC tiene la ventaja de utilizar información e infraestructura del Servicio de Impuestos Internos (SII), lo que disminuye considerablemente los costos administrativos respecto a los programas de transferencias que además deben generar información para otorgar los beneficios ${ }^{33}$. Como consecuencia, se promueve también la formalización de actividades laborales en el sistema tributario.

32 Cabezas (2012) estudia el efecto exclusivamente del Programa de Asignación Social en la oferta laboral. Sus resultados indican que el programa reduce la oferta laboral de las mujeres en $15 \%$ y $12 \%$ para aquellas con y sin pareja respectivamente, y que el componente de Incremento por Inserción Laboral de la Mujer no tiene impacto alguno.

${ }^{33}$ En Estados Unidos se ha estimado que los costos administrativos del EITC son el $0,5 \%$ del monto total de beneficios entregados, mientras que para programas de transferencias tradicionales los costos administrativos representan el 16\%. (Eissa y Hoynes 2011) 
Por último, y como fue mencionado anteriormente, los efectos del crédito tributario al ingreso pueden variar según la fuente de financiamiento que se utilice. Por ejemplo, para no contrarrestar los efectos positivos en la reducción de pobreza y desigualdad, sería necesario que los recursos provengan de la aplicación de impuestos progresivos o neutrales, o en su defecto de la sustitución de otros programas vigentes que tengan objetivos similares a los de un EITC.

Un ejemplo de programas que podrían ser sustituidos por un EITC es el Programa de Asignación Familiar del Instituto de Previsión Social que entrega un monto mensual por cada carga del beneficiario. Al igual que el IEF, el monto es estrictamente creciente con el número de cargas $\mathrm{y}$, si bien está sujeto a la condición de trabajar, otorga beneficios principalmente al sector de asalariados, restringiendo las posibilidades de acceso para los trabajadores independientes que viven en situación de pobreza. Otros ejemplos son los programas pro empleo para beneficiarios del Sistema Chile Solidario, Bonificación a la Contratación de Mano de Obra e Inversión en la Comunidad, que son políticas de empleo directo que subsidian el salario por un período acotado o generan empleo de emergencia en zonas de mayor desempleo. Las evaluaciones a este tipo de programas han sido negativas, principalmente porque son una solución a corto plazo, y no se reflejan en mejorías en los indicadores de empleo o en el nivel de ingreso de sus participantes (Betcherman et al. 2004). Por su parte, el Subsidio al Empleo Joven podría ser absorbido por un programa de crédito tributario, lo que además reduciría el estigma asociado a sus beneficiarios. En total estos programas tienen $\$ 88,4$ mil millones comprometidos para el año 2012 (Dipres 2012).

Resulta relevante mencionar también el Subsidio a la Mujer Trabajadora, que tendrá un presupuesto de \$24.781 millones en 2013, de un total de $\$ 213.886$ millones para el IEF en el año $2013^{34}$. Este programa, al igual que el Subsidio al Empleo Joven, podría formar parte de un programa de crédito tributario al ingreso, ya que además de reducir el estigma de sus beneficiarios, tendría menores costos administrativos a partir del uso de la infraestructura existente del Servicio de Impuestos Internos.

34 http://www.gob.cl/informa/2012/10/05/ministro-lavin-dio-a-conocerlos-programas-y-montos-del-presupuesto-2013.htm accedida el 22 de octubre de 2012. 


\section{Conclusiones}

Durante las últimas décadas los gobiernos de América Latina han utilizado crecientemente programas de transferencias condicionadas como política principal para reducir la pobreza. Si bien estos programas han sido efectivos en reducir la pobreza en el corto plazo, tienen dos falencias importantes: desincentivan el empleo provocando así una trampa de pobreza y generan dependencia del sistema de bienestar social.

En otras regiones del mundo, en cambio, la estrategia para enfrentar altos índices de pobreza y desigualdad ha sido utilizar el sistema impositivo como principal herramienta para ambos fines. Por un lado, un impuesto progresivo al ingreso contribuye a reducir la desigualdad al recaudar en mayor proporción de las personas de más altos ingresos. Por otro lado, transferencias tributarias a las personas de menores ingresos contribuyen no sólo a reducir la desigualdad sino también a disminuir la pobreza. Sin embargo, el mecanismo mediante el cual se hacen estas transferencias es relevante para no generar dependencia ni reducir los incentivos a generar ingresos propios.

Para estos efectos, el mecanismo utilizado ha sido la creación de créditos tributarios por ingreso del trabajo (work income tax credit), y por gastos de las familias como por ejemplo el cuidado de menores (child care tax credit). En aquellos países donde se ha implementado un work income tax credit, como Estados Unidos, Nueva Zelanda e Inglaterra, estos programas se han transformado en la intervención más importante en el combate de la reducción de la pobreza.

En este trabajo se simula la aplicación en Chile de un crédito tributario al ingreso del trabajo basado en el esquema existente en Estados Unidos: el Earned Income Tax Credit (EITC). El objetivo principal de este ejercicio es presentar una alternativa viable a los programas de transferencias condicionadas que se han implementando en el país, como el Chile Solidario (2002) y el Ingreso Ético Familiar (2011). Esta alternativa consiste simplemente en replicar en Chile las experiencias exitosas de otros países e introducir las transferencias vía impuestos a los ingresos del trabajo, que ponen los incentivos correctos para que las personas participen en el mercado laboral y generen ingresos permanentes que les permitan salir de la pobreza.

La simulación realizada otorga beneficios a mujeres solteras de los primeros seis deciles de ingreso, en su mayoría a jefas de familia, 
debido a que estas últimas presentan mayores niveles de pobreza y han sido las principales beneficiarias del programa aplicado en Estados Unidos. Los resultados muestran que en Chile el EITC beneficiaría a 500 mil familias, de las cuales un tercio serían lideradas por mujeres que se insertan al mercado del trabajo como resultado de los incentivos que genera el programa. El beneficio promedio anual estimado es de $\$ 350.000$, alcanzando un máximo de $\$ 663.324$ para mujeres madres solteras con dos o más hijos.

La inserción laboral que genera el EITC mejora de forma importante la focalización de los recursos, ya que quienes deciden trabajar después del programa viven en condiciones mucho más precarias que quienes estaban trabajando antes de la implementación de la política. Es posible que debido al bajo nivel de calificación, estas mujeres no han podido encontrar un trabajo que cubra los costos fijos de salir a trabajar, como el transporte y el cuidado de los niños. De esta forma, el EITC complementa el salario y hace más atractivo el mercado del trabajo para ellas.

Junto con incentivar la participación laboral, el programa es efectivo en reducir la pobreza, medida con el ingreso después de impuestos, que baja de $22,5 \%$ a $20,3 \%$, y también en disminuir la desigualdad, al caer el coeficiente de Gini de 0,512 a 0,504 puntos, rompiendo la trampa de pobreza que generalmente producen los programas de transferencias.

Adicionalmente, los resultados de la simulación muestran que si producto del EITC aumentaran las horas trabajadas de quienes participan en el mercado laboral antes de la implementación del programa, en el largo plazo, a medida que fueran mejorando los ingresos de las participantes, irían cayendo los costos del programa. Esto puede también afectar a otros programas sociales, ya que las beneficiarias del EITC podrían dejar de ser elegibles, y así disminuir la dependencia y los costos del sistema de bienestar social.

La condicionalidad de trabajar limita el apoyo a los más vulnerables entre los pobres y beneficia mayormente a quienes tienen una probabilidad más alta de buscar y encontrar trabajo. Por ello hay que entender este programa como complemento de políticas que consideran subsidios directos no condicionados y especialmente de programas de intermediación laboral que aumenten la probabilidad de que los elegibles encuentren un empleo. 
Es necesario tener en cuenta también que el programa EITC no cubriría a individuos que estén trabajando de manera informal, que no registren ingresos en el SII. Y aunque los niveles de informalidad en Chile son bajos con respecto a otros países latinoamericanos, es justamente en los dos primeros quintiles donde hay un mayor nivel de no-registro de actividades, tanto en asalariados que no están cotizando ( $31 \%$ en el quintil I y $21 \%$ en el quintil II) como en independientes que no registran actividades ( $42 \%$ en el primer quintil y $30 \%$ en el segundo). No obstante, este programa debiera hacer, en el mediano y largo plazo, más atractivo el formalizarse, y en la transición del corto al largo plazo es necesario mantener programas de asistencia social tradicionales para familias de alta vulnerabilidad que pudieran ir acompañados de incentivos a la formalización y ser así la puerta de entrada al EITC.

El costo total en transferencias del crédito tributario propuesto alcanza un valor cercano a los $\$ 170$ mil millones, correspondiente al 0,6\% del presupuesto nacional para el año 2012. Para tener una idea de su magnitud, el año 2012 el Sistema Chile Solidario tiene un presupuesto de $\$ 188$ mil millones y el Programa Ingreso Ético Familiar (IEF) uno de $\$ 81,6$ mil millones. Si bien el monto correspondiente al IEF es la mitad de lo calculado acá para el EITC, el primero considera beneficiar a 170.000 familias, es decir, a un tercio de las potenciales beneficiarias del crédito tributario propuesto, lo que se traduce en un costo unitario por familia superior en $25 \%$ al EITC. En ese sentido la propuesta de un EITC para Chile no sólo genera incentivos a incorporarse y mantenerse en el mercado laboral, reduciendo en el largo plazo la dependencia del sistema de protección social, sino que además tiene costos unitarios sensiblemente menores.

Si bien es difícil de cuantificar, un elemento adicional a tener en cuenta respecto a los costos de un programa como el EITC es que al implementarlo a través del sistema tributario se reducen los gastos de administración y se mejora la focalización. Así lo confirma la evidencia para los países que han implementado un EITC (Eissa y Hoynes 2011) y es razonable esperar para Chile que el SII sea más eficiente en gestionar este programa que el Ministerio de Desarrollo Social, gestionando por ejemplo, el Ingreso Ético Familiar, para el que también se necesita información del Servicio de Impuestos Internos. De hecho, el SII tiene más y mejor información de distintas fuentes respecto a los ingresos de las personas, incluso respecto a quienes no pagan impuestos. En el 2011 
por ejemplo, el SII registró información de ingresos de 8.213 .592 personas naturales. De estos contribuyentes, el $83 \%$ está exento pero el SII tiene información de los ingresos formales de todos ellos.

Adicionalmente a los beneficios cuantificados, es importante mencionar que si bien el EITC no genera impactos inmediatos en los comportamientos relacionados con salud y educación de los beneficiarios, lo cual sí puede ocurrir con un programa de transferencias condicionadas, en el largo plazo sí puede tener efectos relevantes en estas dimensiones debido al mayor ingreso de las familias. De hecho, la evidencia empírica ha destacado el rol del EITC en mejorar las condiciones de vida de los niños y su desempeño escolar (Greenstein y Shapiro 1998, Dahl y Lochner 2010).

Finalmente, en investigaciones futuras sería relevante analizar y explorar potenciales ajustes a la estructura de diseño y elementos asociados a la implementación de un programa como el EITC en Chile, considerando para ello distintas características demográficas y distributivas del país. 


\begin{abstract}
ANEXO
GRÁFICO N ${ }^{\circ}$ A1： COMPARACIÓN EFECTO EITC Y CCT SOBRE LA RESTRICCIÓN PRESUPUESTARIA
\end{abstract}

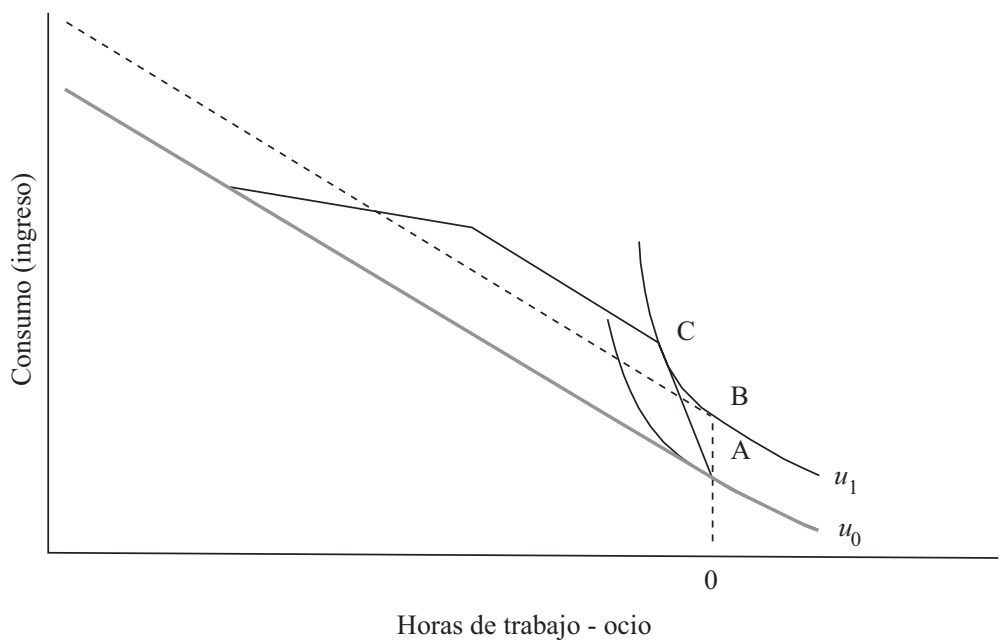

Antes de EITC -..... Después de EITC

Después de CCT

Nota: La situación inicial, sin EITC o programa de transferencia condicionada, para un individuo que no trabaja se encuentra en el punto A, con un nivel de bienestar correspondiente a la curva $u_{0}$. La entrega de una trasferencia condicionada expande el ingreso no laboral y permite a la persona que no trabaja alcanzar un mayor nivel de bienestar, por ejemplo, en la curva $u_{1}$. Si el individuo trabaja inicialmente, la expansión de la restricción presupuestaria tendrá asociado un efecto ingreso que le permitirá aumentar sus horas de ocio (reduciendo sus horas de trabajo) y su nivel de consumo. Por su parte, un individuo que no trabaja antes ni después del EITC se mantendrá en el punto inicial A después de aplicado el crédito, pero si decide insertarse en el mercado laboral, siempre accederá a un nivel de bienestar superior, por ejemplo $u_{1}$. Por lo tanto, para un mismo nivel de bienestar final, $u_{1}$, un programa de transferencias condicionadas permite alcanzar el punto B, y el EITC incentiva el trabajo y hace posible llegar al punto $\mathrm{C}$.

Fuente: Elaboración propia. 
GRÁFICO No A2: PROBABILIDAD PREDICHA DE TRABAJAR DE MUJERES ELEGIBLES (20 Y 45 HORAS)
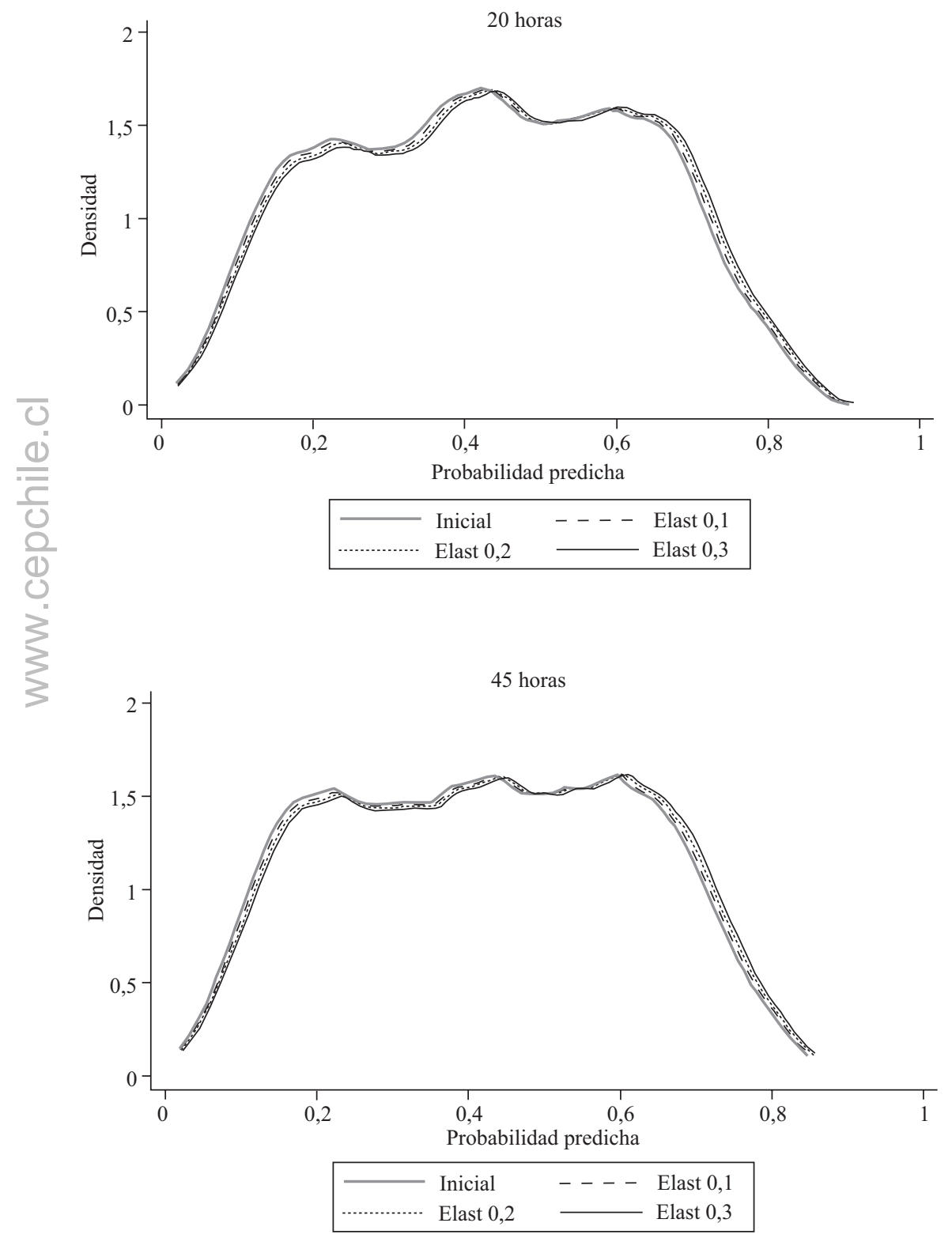

Fuente: Elaboración propia en base a datos de Encuesta Casen 2009. 
TABLA N ${ }^{\circ}$ A1: $\quad$ ECUACIÓN DE SALARIOS PERSONAS ENTRE 18 Y 60 AÑOS (HECKMAN SELECTION)

Logaritmo del salario por hora

Escolaridad (años)

$0,0383 * * *$

$(0,00129)$

Experiencia laboral (edad-años escol-6)

$0,00585 * * *$

$(0,000838)$

Experiencia laboral al cuadrado

$-0,00000442$

$(0,0000169)$

Ingreso otras personas del hogar

$0,000000250^{* * *}$

$(2,78 \mathrm{e}-08)$

Zona (1: urbana)

$0,0373 * * *$

$(0,00944)$

Región Metropolitana

$0,0255 * * *$

$(0,00785)$

Educ. madre: Media

$0,0675 * * *$

$(0,0120)$

Educ. madre: Superior

$0,132 * * *$

$(0,0222)$

Educ. padre: Media

$0,0358 * * *$

$(0,0100)$

Educ. padre: Superior

$0,179 * * *$

$(0,0192)$

Sexo

$0,0852 * * *$

$(0,00721)$

Constante

$2,561 * * *$

$(0,0228)$

Rho

$-0,216^{* * *}$

$(0,0274764)$

Lambda

$-0,0694 * * *$

$(0,0090625)$

Observaciones

132565

Test F

250,3

Errores estándar en paréntesis. * $p<0,10, * * p<0,05, * * * p<0,01$.

Fuente: Elaboración propia en base a datos de Encuesta Casen 2009. 
TABLA N ${ }^{\circ}$ A2: $\quad$ MODELO DE PROBABILIDAD DE PARTICIPACIÓN LABORAL ECUACIÓN DE SELECCIÓN

Participación laboral

Escolaridad (años)

$0,0461 * * *$

$(0,00245)$

Edad

$0,196^{* * *}$

$(0,00426)$

Edad al cuadrado

$-0,00228 * * *$

$(0,0000552)$

Ingreso otras personas del hogar

$-0,000000209^{* * *}$

Zona (1: urbana)

$(4,53 \mathrm{e}-08)$

$0,0423 * *$

$(0,0177)$

Región Metropolitana

$0,152 * * *$

$(0,0185)$

Educ. madre: Media

$0,118 * * *$

$(0,0284)$

Educ. madre: Superior

$0,257 * * *$

$(0,0770)$

0,0176

Educ. padre: Media

$(0,0246)$

0,0456

Educ. padre: Superior

$(0,0625)$

Sexo

$0,925 * * *$

$(0,0163)$

Otros ocupados en el hogar

$-0,253^{* * *}$

$(0,0234)$

$-0,0331^{*}$

$(0,0172)$

$-0,729 * * *$

$(0,0315)$

$-0,0293$

$(0,0219)$

0,0576

Viudo(a)

$(0,0562)$

Separado(a)

$0,276 * * *$

$(0,0302)$

Niños entre 0 y 2 años en el hogar

$0,0437 * *$

$(0,0202)$

Niños entre 3 y 5 años en el hogar

$0,0617 * * *$

$(0,0206)$

$-0,00214$

Niños entre 6 y 12 años en el hogar

$(0,0154)$

Constante

$-4,302 * * *$

$(0,0913)$

Errores estándar en paréntesis. $* p<0,10, * * p<0,05, * * * p<0,01$.

Fuente: Elaboración propia en base a datos de Encuesta Casen 2009. 
TABLA N ${ }^{\circ}$ A3: $\quad$ ESTADÍSTICAS DESCRIPTIVAS

\begin{tabular}{|c|c|c|c|c|c|c|}
\hline \multirow[b]{2}{*}{ Característica } & \multicolumn{3}{|c|}{ Muestra Casen 2009 completa } & \multicolumn{3}{|c|}{ Muestra utilizada en simulación } \\
\hline & Obs. & Media & Desv. est. & Obs. & Media & Desv. est. \\
\hline Menor de 24 años & 244118 & $40 \%$ & $49 \%$ & 230116 & $40 \%$ & $49 \%$ \\
\hline Entre 25 y 34 años & 244118 & $11 \%$ & $31 \%$ & 230116 & $11 \%$ & $31 \%$ \\
\hline Entre 35 y 44 años & 244118 & $14 \%$ & $34 \%$ & 230116 & $14 \%$ & $34 \%$ \\
\hline Entre 45 y 60 años & 244118 & $19 \%$ & $39 \%$ & 230116 & $18 \%$ & $39 \%$ \\
\hline Mayor de 60 años & 244118 & $17 \%$ & $38 \%$ & 230116 & $17 \%$ & $38 \%$ \\
\hline Años de educación & 193763 & 9,1 & 4,3 & 182246 & 9,1 & 4,3 \\
\hline Sin educación & 193763 & $6 \%$ & $23 \%$ & 182246 & $6 \%$ & $23 \%$ \\
\hline $\begin{array}{l}\text { Educación básica - } \\
\text { media incompleta }\end{array}$ & 193763 & $55 \%$ & $50 \%$ & 182246 & $55 \%$ & $50 \%$ \\
\hline Educación media completa & 193763 & $27 \%$ & $44 \%$ & 182246 & $27 \%$ & $44 \%$ \\
\hline Educación superior & 193763 & $13 \%$ & $34 \%$ & 182246 & $13 \%$ & $34 \%$ \\
\hline Estado civil (1: Soltero) & 246924 & $49 \%$ & $50 \%$ & 232772 & $49 \%$ & $50 \%$ \\
\hline Estado civil (1: Casado) & 246924 & $42 \%$ & $49 \%$ & 232772 & $42 \%$ & $49 \%$ \\
\hline Estado civil (1: Viudo) & 246924 & $5 \%$ & $21 \%$ & 232772 & $5 \%$ & $21 \%$ \\
\hline Estado civil (1: Separado) & 246924 & $4 \%$ & $19 \%$ & 232772 & $4 \%$ & $19 \%$ \\
\hline Zona (1: Urbana) & 246924 & $64 \%$ & $48 \%$ & 232772 & $64 \%$ & $48 \%$ \\
\hline Región Metropolitana & 246924 & $20 \%$ & $40 \%$ & 232772 & $20 \%$ & $40 \%$ \\
\hline Ingreso del trabajo por hora & 85.784 & $2.333,5$ & $5.454,5$ & 82.991 & $2.321,4$ & $5.473,7$ \\
\hline $\begin{array}{l}\text { Ingreso familiar per cápita } \\
\text { antes de impuestos }\end{array}$ & 246.924 & $24.774,8$ & $219.286,6$ & 232.772 & $125.459,6$ & $220.510,4$ \\
\hline
\end{tabular}

Fuente: Elaboración propia en base a datos de Encuesta Casen 2009.

TABLA N ${ }^{\circ}$ A4: $\quad$ ESQUEMA DEL EARNED INCOME TAX CREDIT AÑO 1996

\begin{tabular}{ccc}
\hline Ingreso del hogar $(\mathrm{x})$ & Segmento & Crédito $(2$ hijos $)$ \\
$\$ 0-\$ 8.890$ & Primer segmento & $40 \%{ }^{*} \mathrm{x}$ \\
$\$ 8.890-\$ 11.610$ & Fase plana & $\$ 3.556$ \\
$\$ 11.610-\$ 28.495$ & Tercer segmento & $\$ 3.556-21,06 \% *(\mathrm{x}-\$ 11.610)$ \\
\hline Ingreso del hogar $(\mathrm{x})$ & Segmento & Crédito $(1$ hijo $)$ \\
$\$ 0-\$ 6.330$ & Primer segmento & $34 \% * \mathrm{x}$ \\
$\$ 6.330-\$ 11.610$ & Fase plana & $\$ 2.152$ \\
$\$ 11.610-\$ 25.750$ & Tercer segmento & $\$ 2.152-15,98 \% *(\mathrm{x}-\$ 11.610)$ \\
\hline Ingreso del hogar $(\mathrm{x})$ & Segmento & Crédito (sin hijos) \\
$\$ 0-\$ 4.220$ & Primer segmento & $7.65 \% * \mathrm{x}$ \\
$\$ 4.220-\$ 5.280$ & Fase plana & $\$ 323$ \\
$\$ 5.280-\$ 9.500$ & Tercer segmento & $\$ 323-7,65 \% *(\mathrm{x}-\$ 5.280)$ \\
\hline
\end{tabular}

Fuente: Elaboración propia en base a datos de Internal Revenue Service (IRS). http://www.irs.gov. 
TABLA N A5: $\quad$ ESQUEMA DEL EARNED INCOME TAX CREDIT AÑO 2009

\begin{tabular}{|c|c|c|}
\hline Ingreso del hogar (x) & Segmento & Crédito (3 o + hijos) \\
\hline$\$ 0-\$ 12.570$ & Primer segmento & $45 \% * x$ \\
\hline$\$ 12.570-\$ 16.420$ & Fase plana & $\$ 5.657$ \\
\hline$\$ 16.420-\$ 43.279$ & Tercer segmento & $\$ 5.657-21,06 \% *(x-\$ 16.420)$ \\
\hline Ingreso del hogar (x) & Segmento & Crédito (2 hijos) \\
\hline$\$ 0-\$ 12.570$ & Primer segmento & $40 \% * x$ \\
\hline$\$ 12.570-\$ 16.420$ & Fase plana & $\$ 5.028$ \\
\hline$\$ 16.420-\$ 40.295$ & Tercer segmento & $\$ 5.028-21,06 \% *(x-\$ 16.420)$ \\
\hline Ingreso del hogar (x) & Segmento & Crédito (1 hijo) \\
\hline$\$ 0-\$ 8.950$ & Primer segmento & $34 \% * x$ \\
\hline$\$ 8.950-\$ 16.420$ & Fase plana & $\$ 3.043$ \\
\hline$\$ 16.420-\$ 35.463$ & Tercer segmento & $\$ 3.043-15,98 \% *(\mathrm{x}-\$ 16.420)$ \\
\hline Ingreso del hogar (x) & Segmento & Crédito (sin hijos) \\
\hline$\$ 0-\$ 5.970$ & Primer segmento & $7,65 \% * x$ \\
\hline$\$ 5.970-\$ 7.470$ & Fase plana & $\$ 457$ \\
\hline$\$ 7.470-\$ 13.440$ & Tercer segmento & $\$ 457-7,65 \% *(x-\$ 7.470)$ \\
\hline
\end{tabular}

Nota: A partir del año 2009, se hace diferencia entre 2 hijos y 3 o más hijos.

Fuente: Elaboración propia en base a datos de Internal Revenue Service (IRS). http://www.irs.gov.

TABLA N ${ }^{\circ}$ A6: $\quad$ ÍNDICES DE POBREZA ANTES Y DESPUÉS DEL EITC (ESCENARIO H)

\begin{tabular}{lccc}
\hline & $\begin{array}{c}\text { Tasa de } \\
\text { pobreza }\end{array}$ & $\begin{array}{c}\text { Brecha de } \\
\text { pobreza }\end{array}$ & $\begin{array}{c}\text { Brecha de } \\
\text { pobreza }\end{array}$ \\
\hline Antes de EITC & 0,225 & 0,101 & 0,069 \\
& $(0,004)$ & $(0,002)$ & $(0,002)$ \\
Cambio después de EITC & $-0,021^{* * *}$ & $-0,012 * * *$ & $-0,009 * * *$ \\
Escenario C: 45 horas $/ \gamma_{k}=0,3 ; \varepsilon_{k}=0$ & $(0,001)$ & $(0,001)$ & $(0,000)$ \\
Escenario H: 45 horas $/ \gamma_{k}=0,3 ; \varepsilon_{k}=0,1$ & $-0,021^{* * *}$ & $-0,012 * * *$ & $-0,009 * * *$ \\
& $(0,001)$ & $(0,001)$ & $(0,000)$ \\
\hline
\end{tabular}

Nota: Error estándar calculado con el método de bootstrap en paréntesis. ${ }^{*} p<0,10$, $* * p<0,05, * * * p<0,01$.

Fuente: Elaboración propia en base a datos de Encuesta Casen 2009. 
TABLA N ${ }^{\circ}$ A7: $\quad$ INDICADORES DE DESIGUALDAD ANTES Y DESPUÉS DEL EITC (ESCENARIO H)

\begin{tabular}{lcccc}
\hline & $\mathrm{p} 10 / \mathrm{p} 50$ & $\mathrm{p} 75 / \mathrm{p} 25$ & $\mathrm{p} 90 / \mathrm{p} 10$ & Gini \\
& & & & \\
\hline & 0,357 & 2,987 & 9,264 & 0,512 \\
Antes de EITC & $(0,004)$ & $(0,041)$ & $(0,293)$ & $(0,006)$ \\
& & & & \\
Cambio después de EITC & & & \\
Escenario C: 45 horas $/ \gamma_{k}=0,3 ; \varepsilon_{k}=0$ & $0,022^{* * *}$ & $-0,141^{* * *}$ & $-0,695^{* * *}$ & $-0,008^{* * *}$ \\
& $(0,002)$ & $(0,010)$ & $(0,055)$ & $(0,000)$ \\
Escenario H: 45 horas $/ \gamma_{k}=0,3 ; \varepsilon_{k}=0,1$ & $0,022^{* * *}$ & $-0,124^{* * *}$ & $-0,552^{* * *}$ & $0,012^{* * *}$ \\
& $(0,002)$ & $(0,012)$ & $(0,060)$ & $(0,002)$ \\
& & & & \\
\hline
\end{tabular}

Nota: Error estándar calculado con el método de bootstrap en paréntesis. ${ }^{*} p<0,10$, $* * p<0,05, * * * p<0,01$.

Fuente: Elaboración propia en base a datos de Encuesta Casen 2009.

TABLA N ${ }^{\circ}$ A8: $\quad$ ÍNDICES DE POBREZA ANTES Y DESPUÉS DEL EITC (ESCENARIOS B-1 Y C-1)

\begin{tabular}{lccc}
\hline & $\begin{array}{c}\text { Tasa de } \\
\text { pobreza }\end{array}$ & $\begin{array}{c}\text { Brecha de } \\
\text { pobreza }\end{array}$ & $\begin{array}{c}\text { Brecha de } \\
\text { pobreza }\end{array}$ \\
\hline Antes de EITC & 0,225 & 0,101 & 0,069 \\
& $(0,004)$ & $(0,002)$ & $(0,002)$ \\
Cambio después de EITC & & & \\
Escenario B: 20 horas $/ \gamma_{k}=0,3$ & $-0,022^{* * *}$ & $-0,014^{* * * *}$ & $-0,011^{* * *}$ \\
Escenario C: 45 horas $/ \gamma_{k}=0,3$ & $(0,001)$ & $(0,001)$ & $(0,000)$ \\
& $-0,021^{* * *}$ & $-0,012^{* * *}$ & $-0,009^{* * *}$ \\
Escenario B-1: 20 horas $/ \gamma_{k}=0,3$ & $(0,001)$ & $(0,001)$ & $(0,000)$ \\
& $-0,018^{* * *}$ & $-0,011^{* * *}$ & $-0,008^{* * *}$ \\
Escenario C-1: 45 horas $/ \gamma_{k}=0,3$ & $(0,001)$ & $(0,000)$ & $(0,000)$ \\
& $-0,017^{* * *}$ & $-0,009^{* * * *}$ & $-0,006^{* * *}$ \\
& $(0,001)$ & $(0,000)$ & $(0,000)$ \\
\hline
\end{tabular}

Nota: Error estándar calculado con el método de bootstrap en paréntesis. ${ }^{*} p<0,10$, $* * p<0,05, * * * p<0,01$.

Fuente: Elaboración propia en base a datos de Encuesta Casen 2009. 
TABLA $\mathrm{N}^{\circ}$ A9: INDICADORES DE DESIGUALDAD ANTES Y DESPUÉS DE EITC (ESCENARIOS B-1 Y C-1)

\begin{tabular}{lccc}
\hline & $\begin{array}{c}\text { Tasa de } \\
\text { pobreza }\end{array}$ & $\begin{array}{c}\text { Brecha de } \\
\text { pobreza }\end{array}$ & $\begin{array}{c}\text { Brecha de } \\
\text { pobreza }\end{array}$ \\
\hline & & & \\
Antes de EITC & 0,225 & 0,101 & 0,069 \\
Cambio después de EITC & $(0,004)$ & $(0,002)$ & $(0,002)$ \\
Escenario B: 20 horas $/ \gamma_{k}=0,3$ & $0,026^{* * *}$ & $-0,138^{* * *}$ & $-0,765^{* * * *}$ \\
& $(0,002)$ & $(0,010)$ & $(0,056)$ \\
Escenario C: 45 horas $/ \gamma_{k}=0,3$ & $0,022^{* * *}$ & $-0,141^{* * *}$ & $-0,695 * * *$ \\
& $(0,002)$ & $(0,010)$ & $(0,055)$ \\
Escenario B-1: 20 horas $/ \gamma_{k}=0,3$ & $0,019 * * *$ & $-0,124^{* * * *}$ & $-0,592^{* * * *}$ \\
& $(0,002)$ & $(0,009)$ & $(0,050)$ \\
Escenario C-1: 45 horas $/ \gamma_{k}=0,3$ & $0,016^{* * *}$ & $-0,122^{* * *}$ & $-0,536^{* * * *}$ \\
& $(0,002)$ & $(0,009)$ & $(0,052)$ \\
& & & \\
\hline
\end{tabular}

Nota: Error estándar calculado con el método de bootstrap en paréntesis. ${ }^{*} p<0,10$, $* * p<0,05, * * * p<0,01$.

Fuente: Elaboración propia en base a datos de Encuesta Casen 2009. 
TABLA N A10: $\quad$ ESQUEMA PROGRAMA ASIGNACIÓN SOCIAL (DATOS 2011)

Beneficios entregados en base puntaje en Ficha de Protección Social (FPS)

Beneficiario

Beneficio (periodicidad)

Requisito

0-2515 2516-3207 3208-4213

$\begin{array}{lllll}\text { Individual } & \begin{array}{l}\text { Asignación base (mensual) } \\ \text { Niños menores de 6 años }\end{array} & \begin{array}{l}\text { Asignación control niño } \\ \text { sano (mensual) }\end{array} & \$ 6.000 & \$ 4.500 \\ \begin{array}{l}\text { Entre 6 y 18 años } \\ \text { matriculados }\end{array} & \begin{array}{l}\text { Asignación de escolaridad } \\ \text { por matricula (mensual) }\end{array} & \$ 5.000 & \$ 4.000 & \$ 3.000 \\ \begin{array}{c}\text { Entre 6 y 18 años con } \\ \text { un 85\% de asistencia }\end{array} & \begin{array}{l}\text { Asignación de escolaridad } \\ \text { por asistencia (mensual) }\end{array} & \$ 5.000 & \$ 4.000 & \$ 3.000 \\ \begin{array}{c}\text { Entre 5 } 5^{\circ} \text { Básico y } \\ 4^{\circ} \text { Medio en el 15\% de } \\ \text { los mejores alumnos }\end{array} & \begin{array}{l}\text { Asignación por logro } \\ \text { escolar (anual) }\end{array} & & & \\ & & \$ 50.000 & & \end{array}$

Beneficios entregados en base a Renta Bruta $(X)$

$\begin{array}{ccccc}\text { Beneficiario } & \begin{array}{c}\text { Beneficio } \\ \text { (periodicidad) }\end{array} & \$ 1-\$ 172.000 & \begin{array}{c}\text { Requisito } \\ \$ 172.001-\$ 215.000\end{array} & \$ 215.001-\$ 387.000\end{array}$

$\begin{array}{llll}\begin{array}{l}\text { Mujeres mayores } \\ \text { de } 18 \text { que se }\end{array} & \begin{array}{l}\text { Incremento por } \\ \text { inserción laboral } \\ \text { inserten al } \\ \text { mercado laboral la mujer }\end{array} \\ \begin{array}{c}\text { (anual y se entrega } \\ \text { una sola vez) }\end{array} & 30 \% * \mathrm{X} & \$ 51.600 & \$ 51.600-30 \% *(\mathrm{X}-\$ 215.000)\end{array}$

Nota: Los bonos adicionales por control de niño sano y educación se calculan de forma mensual pero se pagan una o dos veces al año.

Fuente: Elaboración propia en base a información del Ministerio de Desarrollo Social. www.ministeriodesarrollosocial.gob.cl. 


\section{REFERENCIAS}

Acero, C. y G. Cabezas (2011). "Effects of the Ethical Family Income on Labor Participation, Income Distribution and Poverty". Carlos M. Urzúa (ed.), Microsimulation Models for Latin America. Mexico City: ITESM Instituto Tecnológico y de Estudios Superiores de Monterrey.

Betcherman, G., K. Olivas y A. Dar (2004). "Impacts of Active Labor Market Programs: New Evidence from Evaluations with Particular Attention to Developing and Transition Countries". Social Discussion Paper Series $\mathrm{N}^{\mathrm{o}}$ 0402, Banco Mundial.

Bouillon, P. y L. Tejerina (2007). "Do We Know What Works?: A Systematic Review of Impact Evaluations of Social Programs in Latin America and the Caribbean". Inter-American Development Bank (IDB).

Cabezas, G. (2012). "Efecto del Programa Asignación Social sobre la oferta laboral femenina". Tesis para optar al grado de Master of Applied Economics otorgado por Georgetown University y al grado de Magíster en Economía Aplicada a Políticas Públicas de la Universidad Alberto Hurtado. Profesor guía: Marcela Perticará.

Cantallops, J., M. Jorrat y D. Sherman (2007). "Equidad Tributaria en Chile: Un Nuevo Modelo para Evaluar Alternativas de Reforma", Cepal.

Caspersen, E. y G. Metcalf (1994). "Is a Value Added Tax Regressive? Annual Versus Lifetime Incidence”. National Tax Journal 47 (4).

Chetty, R. y E. Sáez (2009). “Teaching the Tax Code: Earnings Responses to an Experiment with EITC Recipients". NBER Working Papers 14836, National Bureau of Economic Research, Inc.

Congressional Budegt Office (2005). "Effective Marginal Tax Rates on Labor Income".

Dahl, G. y L. Lochner (2010). "The Impact of Family Income on Child Achievement: Evidence from the Earned Income Tax Credit". University of Western Ontario, CIBC Centre for Human Capital and Productivity. Working Papers 20105.

De Hoyos, R. (2006). "Structural Modelling of Female Labour Participation and Occupation Decisions". Cambridge Working Papers in Economics 0611, Faculty of Economics, University of Cambridge.

Decoster, A., J. Loughrey, C. O’Donoghue y D. Verwerft (2010). "How Regressive Are Indirect Taxes? A Microsimulation Analysis for Five European Countries". Journal of Policy Analysis and Management, Vol. 29 (2): 326-350.

Dickert, S., S. Hauser y J. K. Scholz (1995). “The Earned Income Tax Credit and Transfer Programs: A Study of Labor Market and Program Participation”. En James M. Poterba (ed.), Tax Policy and the Economy, Vol. 9, MIT Press.

Dirección de Presupuestos (2012). Resumen Presupuesto de Programas. http:// www.dipres.gob.cl/574/propertyvalue-15146.html.

Eissa, N. y H. Hoynes (2004). "Taxes and the Labor Market Participation of Married Couples: The Earned Income Tax Credit". Journal of Public Economics, Vol. 88 (9-10): 1931-1958, August 2004. 
(2006). "Behavioral Responses to Taxes: Lessons from the EITC and Labor Supply". Tax Policy and the Economy, Vol. 20: 74-110. MIT Press.

(2008). "Redistribution and Tax Expenditures: The Earned Income Tax Credit". NBER Working Paper No. 14307, National Bureau of Economic Research, Inc.

(2011). "Redistribution and Tax Expenditures: The Earned Income Tax Credit". National Tax Journal, June 2011, 64 (2, Part 2): 689-730.

Eissa, N., H. Kleven y C. Kreiner (2008). "Evaluation of Four Tax Reforms in the United States: Labor Supply and Welfare Effects for Single Mothers". Journal of Public Economics, April 2008, 92 (3-4): 795-816.

Eissa, N. y J. Liebman (1996). "Labor Supply Response to the Earned Income Tax Credit". Quarterly Journal of Economics, CXI, 605-637.

Eissa, N. y A. Nichols (2005). "Tax-Transfer Policy and Labor Market Outcomes". Washington, DC: The Urban Institute.

Evers, M., R. de Mooji y D. van Vuuren (2005). "What Explains the Variation in Estimates of Labour Supply Elasticities?”. CESifo Working Paper No. 1633 Category 4: Labour Markets December 2005.

Fiszbein, A. y N. Schady (2009). "Conditional Cash Transfer”. Banco Mundial.

Foster, J., J. Greer y E. Thorbecke (1984). “A Class of Descomi'posable Poverty Measures”. Econometrica, Vol. 52 (3): 761-762.

Greenstein, R. e I. Shapiro (1998). "New Research Findings on the Effects of the Earned Income Tax Credit". Washington, DC: Center on Budget and Policy Priorities.

Grogger, J. (2003). "The Effects of Time Limits, the Eitc, and Other Policy Changes on Welfare Use, Work, and Income Among Female-Headed Families". The Review of Economics and Statistics, May 2003, 85 (2): 394-408.

Heckman, J. (1979). "Sample Selection Bias as a Specification Error". Econometrica 47 (1): 153-61.

Holt, S (2006). "The Earned Income Tax Credit Age 30: What we Know". The Brookings Institution, http://www.brookings.edu/ reports/2006/02childrenfamilies_holt.aspx.

Hotz, J. y J. Scholz (2003). "The Earned Income Tax Credit”. En R. Moffitt (editor), Means-Tested Transfer Programs in the United States. Chicago: University of Chicago Press, pp. 141-198.

Keane, M. y R. Moffitt (1998). "A Structural Model of Multiple Welfare Program Participation and Labor Supply". International Economic Review, 39 (3): 553-589.

Leahy, E., S. Lyons y R. Tol (2011). "The Distributional Effects of Value Added Tax in Ireland". The Economic and Social Review 42 (2): 213-235.

Leigh, A. (2010). "Who Benefits from the Earned Income Tax Credit? Incidence Among Recipients, Coworkers and Firms". The B. E. Journal of Economic Analysis \& Policy, Vol. 10 (1), Article 45.

Meyer, B. (2002). "Labor Supply at the Extensive and Intensive Margins: The EITC, Welfare, and Hours Worked". American Economic Review, May, 92 (2): 373-379. 
(2007). "The U.S. Earned Income Tax Credit, its Effects, and Possible Reforms". Swedish Economic Policy Review, 14 (0720): 55-80.

Meyer, B. y D. Rosenbaum (1999). "Welfare, the Earned Income Tax Credit, and the Labor Supply of Single Mothers". NBER Working Paper 7363.

Ministerio de Desarrollo Social (2011). "Lanzamiento Asignación Social". www.emol.com/documentos/archivos/2011/.../20110323144436.pdf.

Mirrlees, J. (1971). "An Exploration in the Theory of Optimum Income Taxation". Review of Economics Studies 38: 175-208.

(1976). "Optimal Tax Theory: A Synthesis". Journal of Public Economics 6: 327-358.

Mirrlees, J., S. Adam, T. Besley, R. Blundell, S. Bond, R. Chote, M. Gammie, P. Johnson, G. Myles y J. Poterba (eds.) (2010). Dimensions of Tax Design: The Mirrlees Review. Oxford: Oxford University Press for Institute for Fiscal Studies.

Neumark, D y W. Wascher (2001). "Using the EITC to Help Poor Families: New Evidence and a Comparison with the Minimum Wage". National Tax Journal 54, pp. 281-317.

Rothstein, J. (2005). "The Mid-1990s EITC Expansion: Aggregate Labor Supply Effects and Economic Incidence". Mimeo, Princeton University. (2009). "Is the EITC as Good as an NIT? Conditional Cash Transfers and Tax Incidence". Working Papers 1160, Princeton University, Department of Economics, Center for Economic Policy Studies.

Sáez, E. (2002). "Optimal Income Transfer Programs: Intensive versus Extensive Labor Supply Responses". Quarterly Journal of Economics, 117 (2002), 1039-1073.

Triest, R. (1990). "The Effect of Income Taxation on Labor Supply in the United States". The Journal of Human Resources, Vol. 25 (3), Special Issue on Taxation and Labor Supply in Industrial Countries (Summer, 1990): 491-516.

Wu, X., J. Perloff y A. Golan (2006). "Effects of Taxes and other Government Policies on Income Distribution and Welfare". Institute for Research on Labor and Employment. Working Paper Series 328, Institute of Industrial Relations, UC Berkeley. 